BMC

Genomics

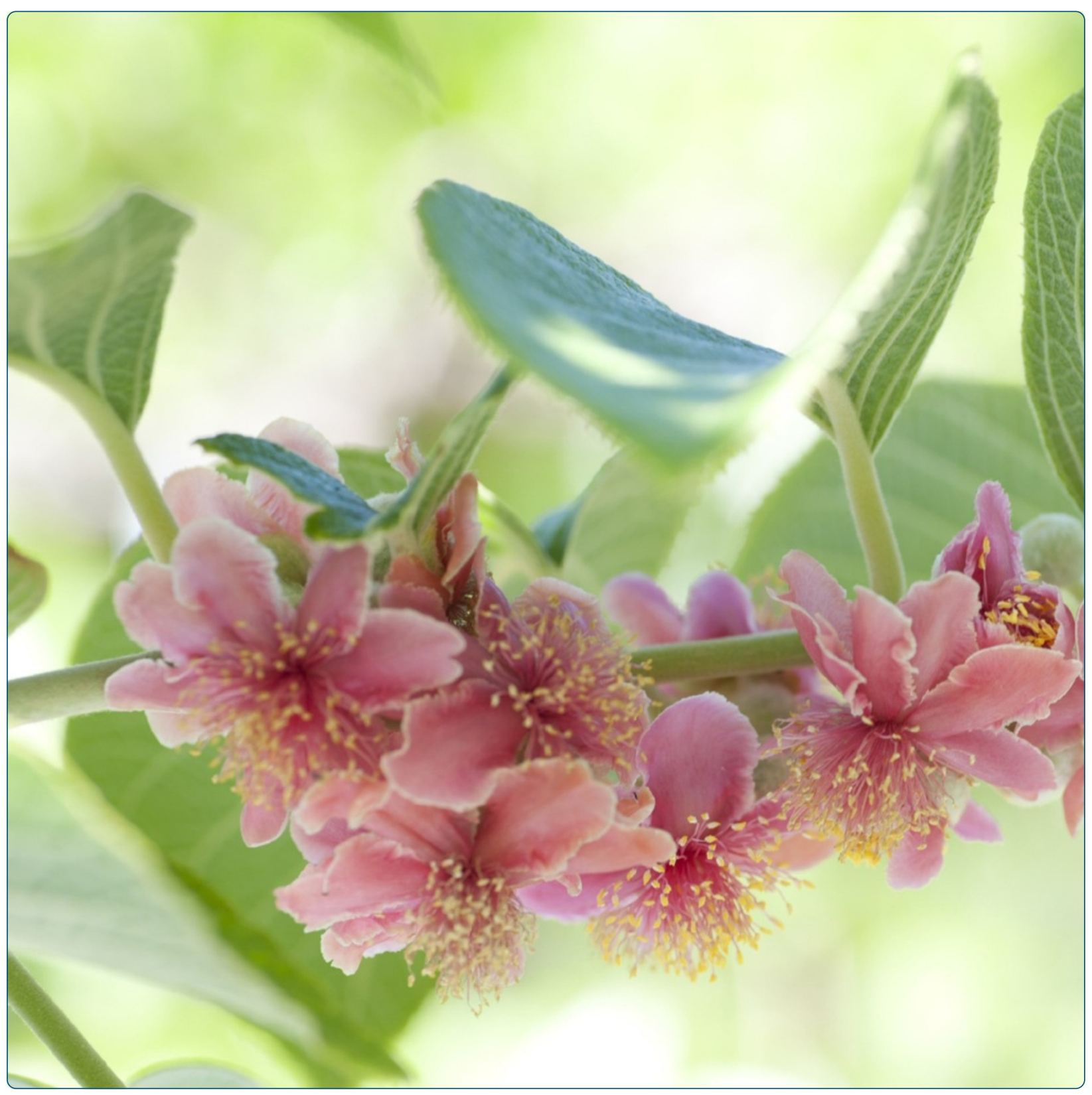

\title{
An R2R3 MYB transcription factor determines red petal colour in an Actinidia (kiwifruit) hybrid population
}

Fraser et al. 


\title{
An R2R3 MYB transcription factor determines red petal colour in an Actinidia (kiwifruit) hybrid population
}

Lena G Fraser ${ }^{1 *}$, Alan G Seal ${ }^{2}$, Mirco Montefiori ${ }^{1}$, Tony K McGhie ${ }^{3}$, Gianna K Tsang ${ }^{1}$, Paul M Datson ${ }^{1}$, Elena Hilario ${ }^{1}$, Hinga E Marsh², Juanita K Dunn², Roger P Hellens', Kevin M Davies ${ }^{3}$, Mark A McNeilage ${ }^{1}$, H Nihal De Silva ${ }^{1}$ and Andrew C Allan ${ }^{1}$

\begin{abstract}
Background: Red colour in kiwifruit results from the presence of anthocyanin pigments. Their expression, however, is complex, and varies among genotypes, species, tissues and environments. An understanding of the biosynthesis, physiology and genetics of the anthocyanins involved, and the control of their expression in different tissues, is required. A complex, the MBW complex, consisting of R2R3-MYB and bHLH transcription factors together with a WD-repeat protein, activates anthocyanin 3-O-galactosyltransferase (F3GT1) to produce anthocyanins. We examined the expression and genetic control of anthocyanins in flowers of Actinidia hybrid families segregating for red and white petal colour.

Results: Four inter-related backcross families between Actinidia chinensis Planch. var. chinensis and Actinidia eriantha Benth. were identified that segregated 1:1 for red or white petal colour. Flower pigments consisted of five known anthocyanins (two delphinidin-based and three cyanidin-based) and three unknowns. Intensity and hue differed in red petals from pale pink to deep magenta, and while intensity of colour increased with total concentration of anthocyanin, no association was found between any particular anthocyanin data and hue. Real time qPCR demonstrated that an R2R3 MYB, MYB110a, was expressed at significant levels in red-petalled progeny, but not in individuals with white petals.

A microsatellite marker was developed that identified alleles that segregated with red petal colour, but not with ovary, stamen filament, or fruit flesh colour in these families. The marker mapped to chromosome 10 in Actinidia. The white petal phenotype was complemented by syringing Agrobacterium tumefaciens carrying Actinidia 355:: MYB110a into the petal tissue. Red pigments developed in white petals both with, and without, co-transformation with Actinidia bHLH partners. MYB110a was shown to directly activate Actinidia F3GT1 in transient assays.
\end{abstract}

Conclusions: The transcription factor, MYB110a, regulates anthocyanin production in petals in this hybrid population, but not in other flower tissues or mature fruit. The identification of delphinidin-based anthocyanins in these flowers provides candidates for colour enhancement in novel fruits.

Keywords: Actinidia, Kiwifruit, Anthocyanin, MYB, Transcription factor, Colour

\footnotetext{
* Correspondence: lena.fraser@plantandfood.co.nz

${ }^{1}$ The New Zealand Institute for Plant \& Food Research Limited, 120 Mt. Albert Road, Auckland 1142, New Zealand

Full list of author information is available at the end of the article
} 


\section{Background}

Flavonoids are a large, diverse group of plant phenolic compounds involved in a variety of biological responses to the biotic and abiotic environment, such as disease resistance, seed dormancy, pigmentation of flowers and fruits, protection against UV-B damage, and reduction of insect and mammalian herbivory [1,2]. The anthocyanin pigments that are produced by a branch of the flavonoid pathway are important as insect and animal attractants, for pollination and seed dispersal. However, they are also of increasing importance as quality traits in commercial fruit crops, not only for the colours they produce but also for their potential human health benefits. A wide range of cell culture experiments, animal trials and epidemiological studies have linked intake of anthocyanins with reduced risk for a range of health problems, including heart disease, cancer, diabetes and degenerative conditions such as Alzheimer's disease [3-6].

As flavonoids contribute to many different functions at various times in a plant, a complex system of regulation is required to direct temporal and spatial production in response to developmental and environmental stimuli. The primary point of regulation of flavonoid production occurs at the transcriptional level, through activation or repression of the biosynthetic genes by a conserved group of transcription factors (TFs). In all plants studied to date, activation of the anthocyanin pathway is through a 'MBW complex' consisting of R2R3-MYB and bHLH TFs and a WD-repeat (WDR) protein that may facilitate protein-protein interactions [7-9]. In addition to the MYBs that are part of the activation complex, distinct R2R3- and R3-MYBs with a repressive action have been identified that may allow for feedback modulation of the amount of anthocyanin produced [10-14].

Based on previous studies, it seems likely that variation in activity of the R2R3-MYB genes is key to determining the spatial and temporal patterning of anthocyanin production in most plant species $[15,16]$. The R2R3-MYB and bHLH families of transcription factors are two of the largest in plants, with 139 and 162 members respectively, in Arabidopsis [17]. The R2R3-MYBs that activate anthocyanin biosynthesis have diverged during evolution to control anthocyanin production only in response to specific stimuli or with a high degree of spatial specificity. R2R3-MYB genes have been identified that are associated with activating anthocyanin biosynthesis in flowers, fruits, or vegetative tissues [11,15,16,18-20]. Genetic changes affecting individual members of the R2R3-MYB or bHLH gene families can thus result in tissue-specific changes in anthocyanin production. The identification of the R2R3-MYB and bHLH genes associated with anthocyanin production in a particular species is therefore an important step towards elucidating the genetic control of pigmentation in that species.

Petals of the kiwifruit species Actinidia chinensis Planch. var. chinensis are white or cream, shading to apricot during senescence, while those of $A$. eriantha Benth. are typically pink or red, although a white-flowered form has been found in China [21]. $F_{1}$ hybrids from crosses between tetraploid $A$. chinensis var. chinensis and $A$. eriantha are known to produce red flowers [22,23]. Zhang et al. (2010) studied the segregation of flower colour among 22 seedlings from a cross between hexaploid $A$. chinensis var. deliciosa and a diploid male $\mathrm{F}_{1}$ hybrid between diploid $A$. eriantha and tetraploid $A$. chinensis var. chinensis [24]. The seedlings could be broadly classified into those with red and those with white-coloured petals, suggesting the action of a small number of genes. However, because of the complexity of the pedigree, the small number of seedlings and the presence of intermediate types with red and white petals, no firm conclusions could be drawn about the inheritance of petal colour. Fan et al. (2004) studied the flower colour of 26 seedlings obtained from a cross between hexaploid $A$. chinensis var. deliciosa and diploid $A$. eriantha [25]. Female seedlings all had white flowers while the male seedlings had red, white or peach and white flowers. The authors reached no conclusion regarding inheritance of petal colour.

In this paper, we report the results from a study of petal colour in two families of (A. eriantha $\mathrm{x} A$. chinensis) $\mathrm{x}$ A. chinensis var. chinensis (EACK $\mathrm{x} C \mathrm{CK}$ ) hybrids and two families of A. chinensis var. chinensis $\mathrm{x}$ A. eriantha) $\mathrm{x}$ A. chinensis var. chinensis (CKEA $\mathrm{x}$ CK) hybrids (Additional files 1,2). The scale of the population and its genetic structure, and the ability to access a large Expressed Sequence Tag (EST) database for Actinidia species in which to seek candidate regulatory genes, enabled us to examine the genetic control of petal colour in these hybrids [26].

\section{Results}

The phenotyping of petal colour of the four backcross families

The ratio of vines with red or white petals in each of the four families was not significantly different from 1:1, and there were no significant differences in petal colour ratio among families $(P=0.382-0.749)$ (Table 1$)$, or between sexes within families $(P=0.109-1.000)$. This result would suggest that, in these hybrid families, petal colour inheritance is a monogenic dominant trait.

Among progeny vines with red petals, some variation was observed in the intensity of red colouration between vines and many had much darker red petals than those of their $\mathrm{F}_{1}$ hybrid parent or $A$. eriantha grandparent (Figure 1). Intensity of colour usually declined from the 
Table 1 Phenotypes of four Actinidia hybrid backcross families

\begin{tabular}{|c|c|c|c|c|c|c|c|}
\hline Family & Red male & White male & Red female & White female & Total Red & Total White & $P$ value $\mathrm{H} \_\mathrm{O}:$ ratio $=1: 1$ \\
\hline 1 & 26 & 24 & 16 & 22 & 42 & 46 & 0.749 \\
\hline 2 & 12 & 14 & 12 & 16 & 24 & 30 & 0.497 \\
\hline 3 & 10 & 14 & 10 & 13 & 20 & 27 & 0.382 \\
\hline 4 & 29 & 25 & 19 & 17 & 48 & 42 & 0.598 \\
\hline Total & 77 & 77 & 57 & 68 & 134 & 145 & 0.550 \\
\hline
\end{tabular}

Segregation for petal colour and gender in two families of backcross seedlings of ( $A$. eriantha $\mathrm{x}$ A. chinensis var. chinensis) $\mathrm{x}$ A. chinensis var. chinensis (families 1 and 2$)$, and two families of backcross seedlings of $(A$. chinensis var. chinensis $\times A$. eriantha) $\times A$. chinensis var. chinensis (families 3 and 4$)$. The ratio of red to white petal colour of approximately 1:1 suggests that in petals of these hybrid families colour inheritance is a monogenic dominant trait.

base of the petal to the fringe. However, there was very little variation between flowers within each vine.

Of 101 vines scored for the colour of the ovary pericarp tissue, only 11 had red colouration, of which four showed intense red colour. These 11 vines all belonged to the same family (family 1 ) and thus had the same parentage. Ovary colour was independent of petal colour. Stamen filament colour was also independent of petal and ovary colour. Red filaments were found with both red or white petals, and red or green ovaries, in all combinations in female progeny, and with red or white petals in males (Additional file 3).
Of 88 female vines screened for fruit flesh colour, only four had fruit with some red colouration and the red colour was faint and confined to the area around the core towards the pedicel end of the fruit. There was no obvious association between red colour in the ovary, petals, filaments or fruit flesh.

\section{Quantification and identification of anthocyanins and flavonols extracted from red petals of individual genotypes}

In all four families there was variation in hue among the red-petalled genotypes (Figure 2). As dihydroxyflavonols

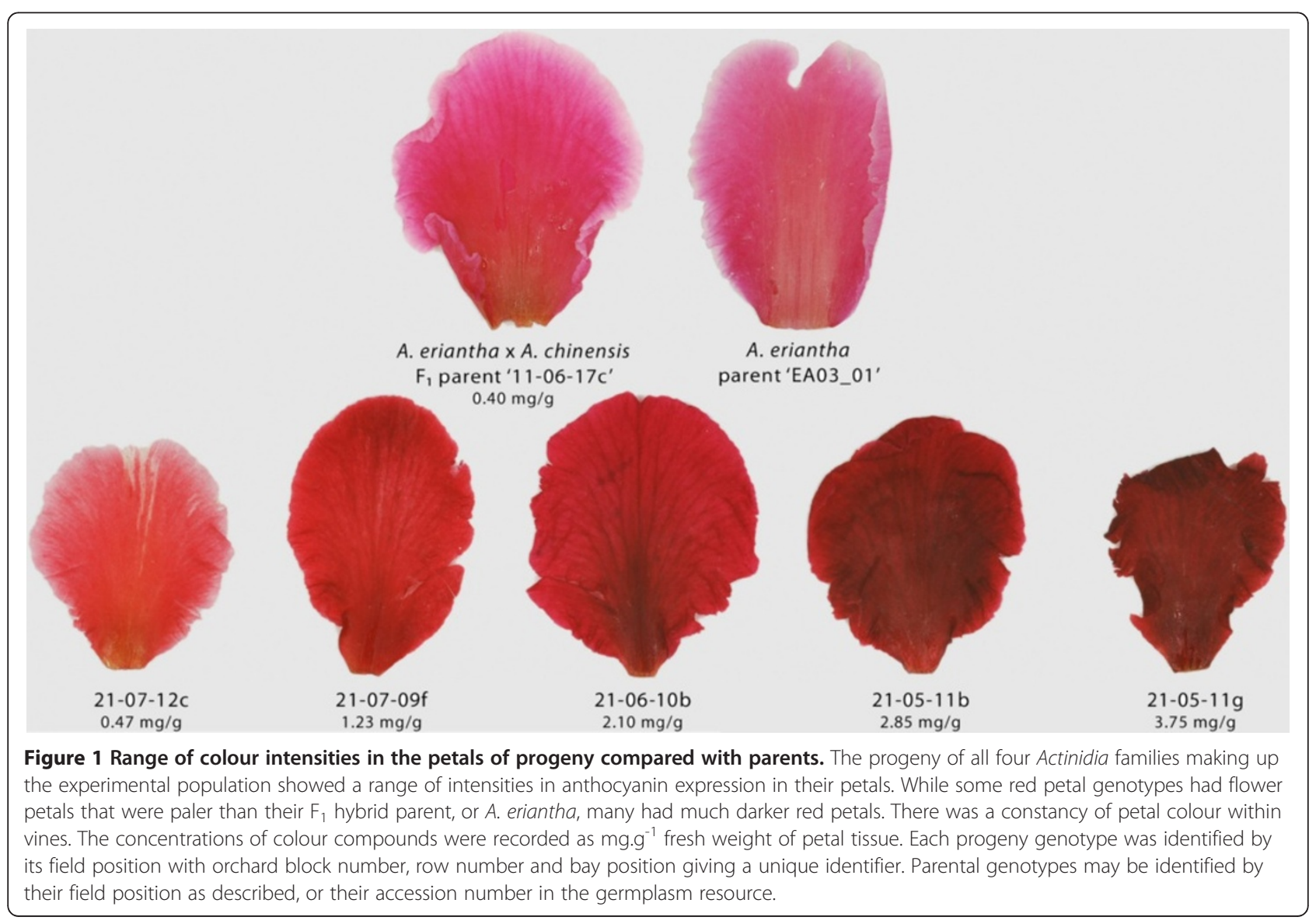



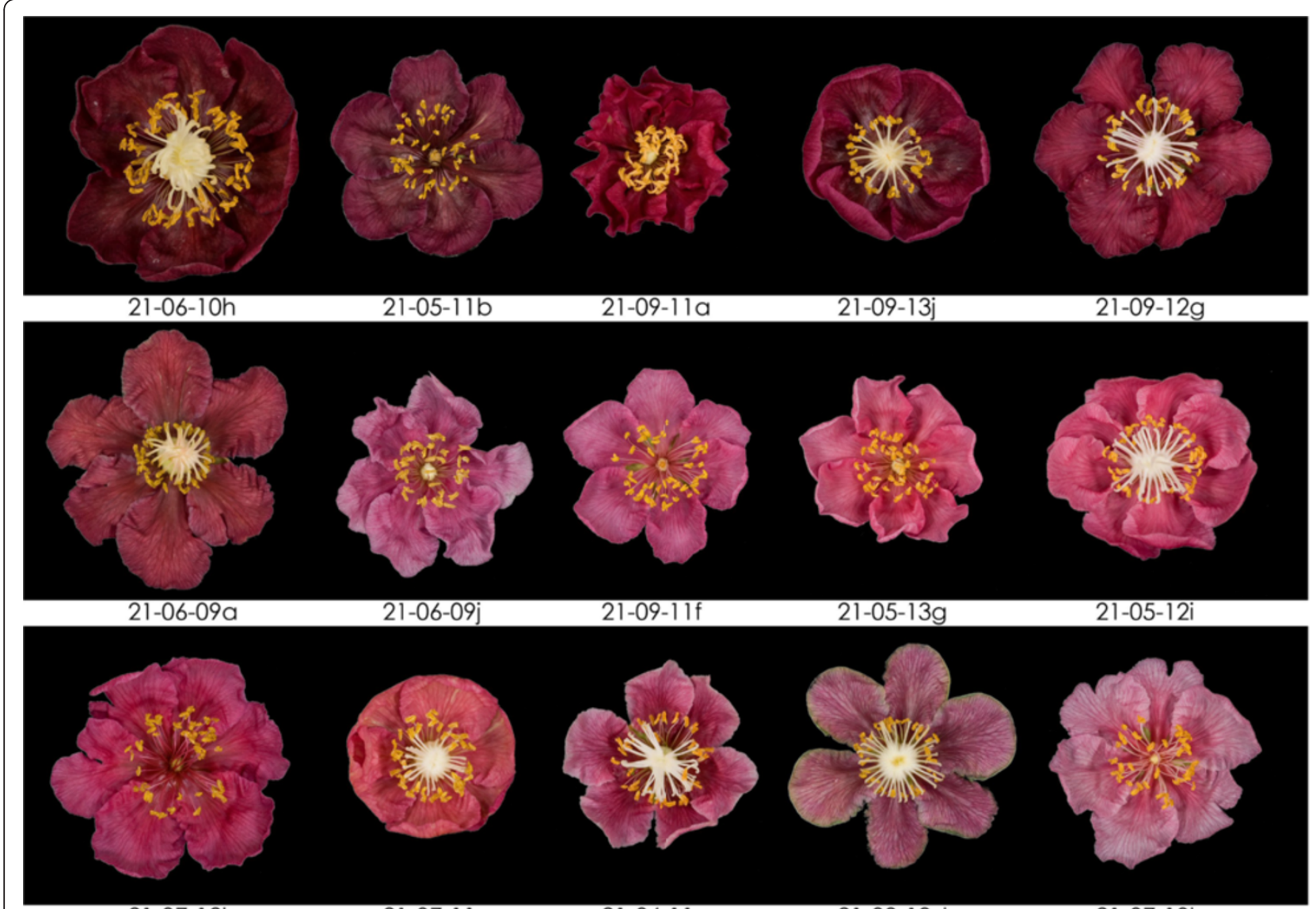

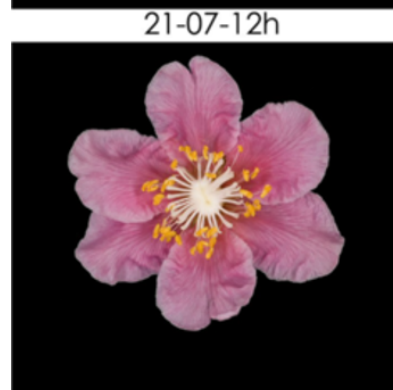

21-06-11

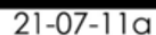

$21-06-11 c$

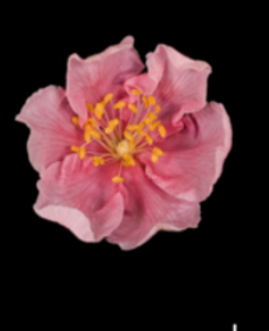

21-08-11a 21-06-12b

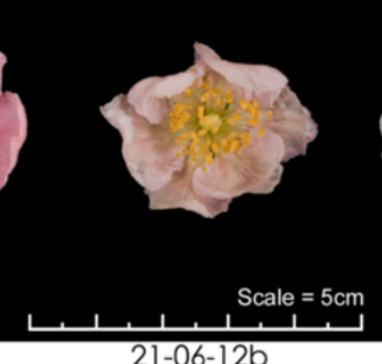

.

$21-09-13 d$

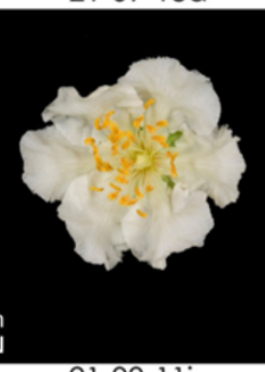

21-09-11j
$21-07-10 b$

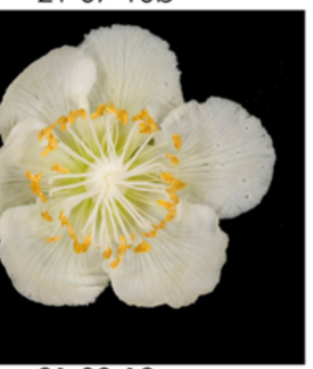

$21-09-12 e$

Figure 2 Colour intensity and hue variation of petals among progeny genotypes. Actinidia progeny segregating for red and white petal colour within all four families showed significant variation among genotypes in both colour intensity and hue of the flower petals. The red flowers ranged from a pale pink to a deep magenta. The unique genotype identity of field position is shown below a typical flower of each.

are substrates for production of flavonols, proanthocyanidins and anthocyanins, and because flavonols are known to act as co-pigments affecting hue, the amounts of these compounds were assayed at the same time as the anthocyanins (Figure 3, Additional file 4).

Eight anthocyanins were detected in red petal tissue. Two of these were classified as delphinidin-based and three as cyanidin-based, while the identities of three anthocyanins that were present in small quantities were not determined (Anthocyanin Unknown, Acn-Unk1 to Acn-Unk3). The concentration of all compounds was recorded as $\mathrm{mg} \cdot \mathrm{g}^{-1}$ fresh weight of petal tissue. The delphinidin-based anthocyanins, delphinidin 3-O-(xylosyl) galactoside (dp-xylgal) and delphinidin 3-O-galactoside (dp-gal), were present in only 40 of the 134 genotypes sampled, and family 2 progeny did not contain delphinidin compounds. Families 1, 3 and 4 had 11 genotypes with dp-xylgal only and 24 genotypes with dp-gal only, while family 1 also had five genotypes with both delphinidins (Additional file 4). The three cyanidin-based anthocyanins identified were cyanidin 3-O-(xylosyl)galactoside (cy-xylgal), cyanidin 3-O-galactoside (cy-gal) and cyanidin 3-O-glucoside (cy-glu). When summed, anthocyanins ranged in concentration from $0.11 \mathrm{mg} . \mathrm{g}^{-1}$ to $3.73 \mathrm{mg} . \mathrm{g}^{-1}$, 


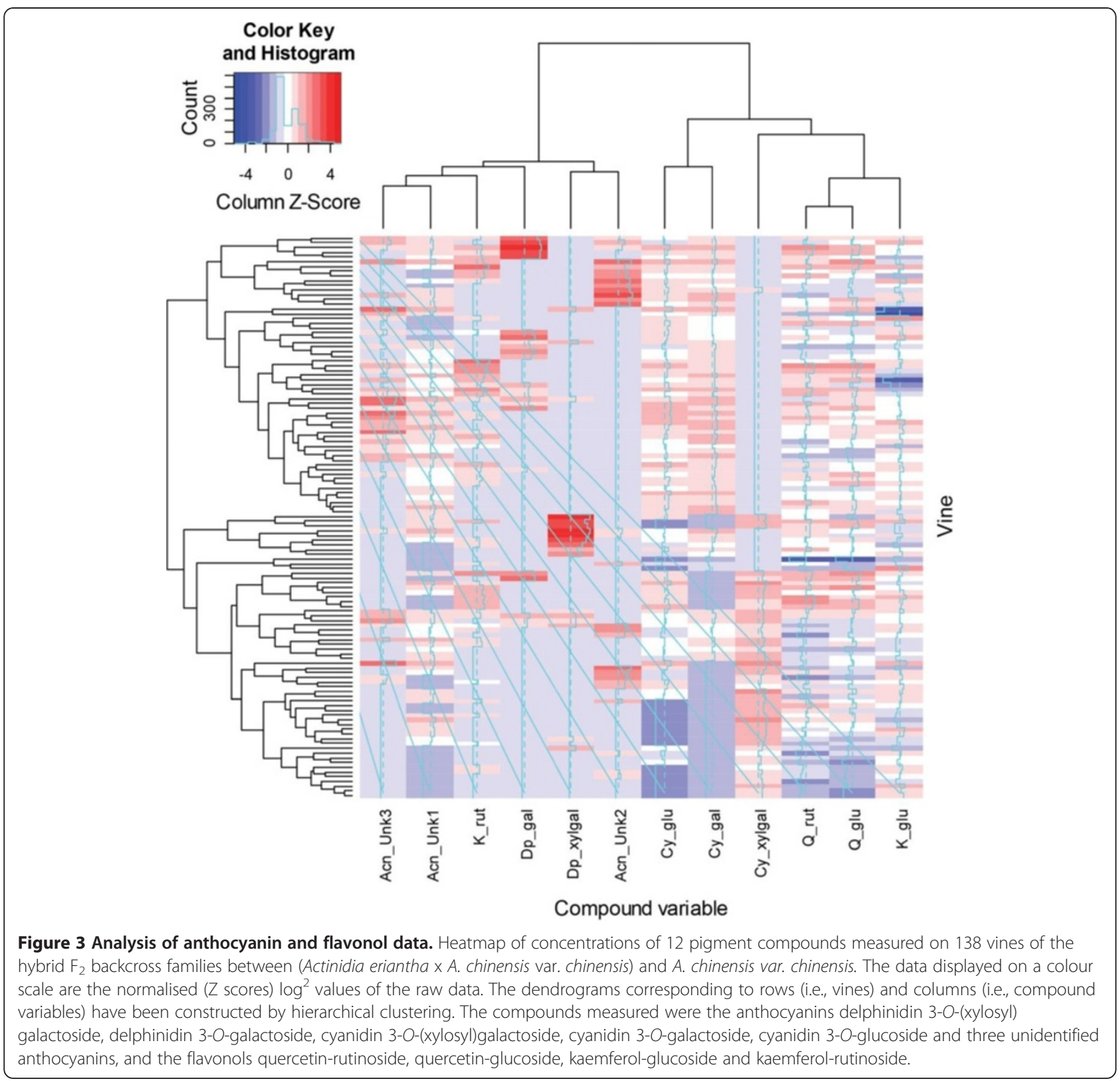

with the higher concentrations reflected in the depth of petal colour. Cy-gal and cy-glu were the two most commonly occurring identified anthocyanins, and Acn-Unk1 also occurred in most genotypes but in very low concentrations from 0.01 to $0.08 \mathrm{mg} \cdot \mathrm{g}^{-1}$. Cy-gal and cy-glu generally occurred in the same genotypes, but the concentrations of cy-glu were significantly lower than those of cy-gal (Additional file 4). Genotypes were recorded as having from one anthocyanin present, either cy-xylgal or cy-gal, to having up to all eight anthocyanins present. Among the chromatograms recorded, four patterns of anthocyanin concentration were found (Figure 4). In Figure 4-traces 1, 2 and 3 respectively, the anthocyanins identified in greatest concentration were 1: cy-gal and cy-glu; 2: cy-xylgal and cy-glu; and 3: cy-gal and dpgal. In the fourth pattern multiple anthocyanins were present, with cy-xylgal and cy-gal dominating the anthocyanin profiles.

Four flavonols were assayed as potential co-pigments. Quercetin-rutinoside (q-rut), quercetin-glucoside (q-glu) and kaemferol-glucoside (k-glu) were present in all genotypes. Concentrations ranged from $0.07 \mathrm{mg} . \mathrm{g}^{-1}$ to 2.02 (q-rut), 3.39 (q-glu) and 6.13 (k-glu) $\mathrm{mg}^{-\mathrm{g}^{-1}}$ of petal tissue. Kaemferol-rutinoside (k-rut) was present in 60 of the 134 genotypes, and at much lower concentrations between 0.02 and $0.33 \mathrm{mg} \cdot \mathrm{g}^{-1}$. There was no apparent association between the concentration of flavonol present and the perceived hue of the petals. 


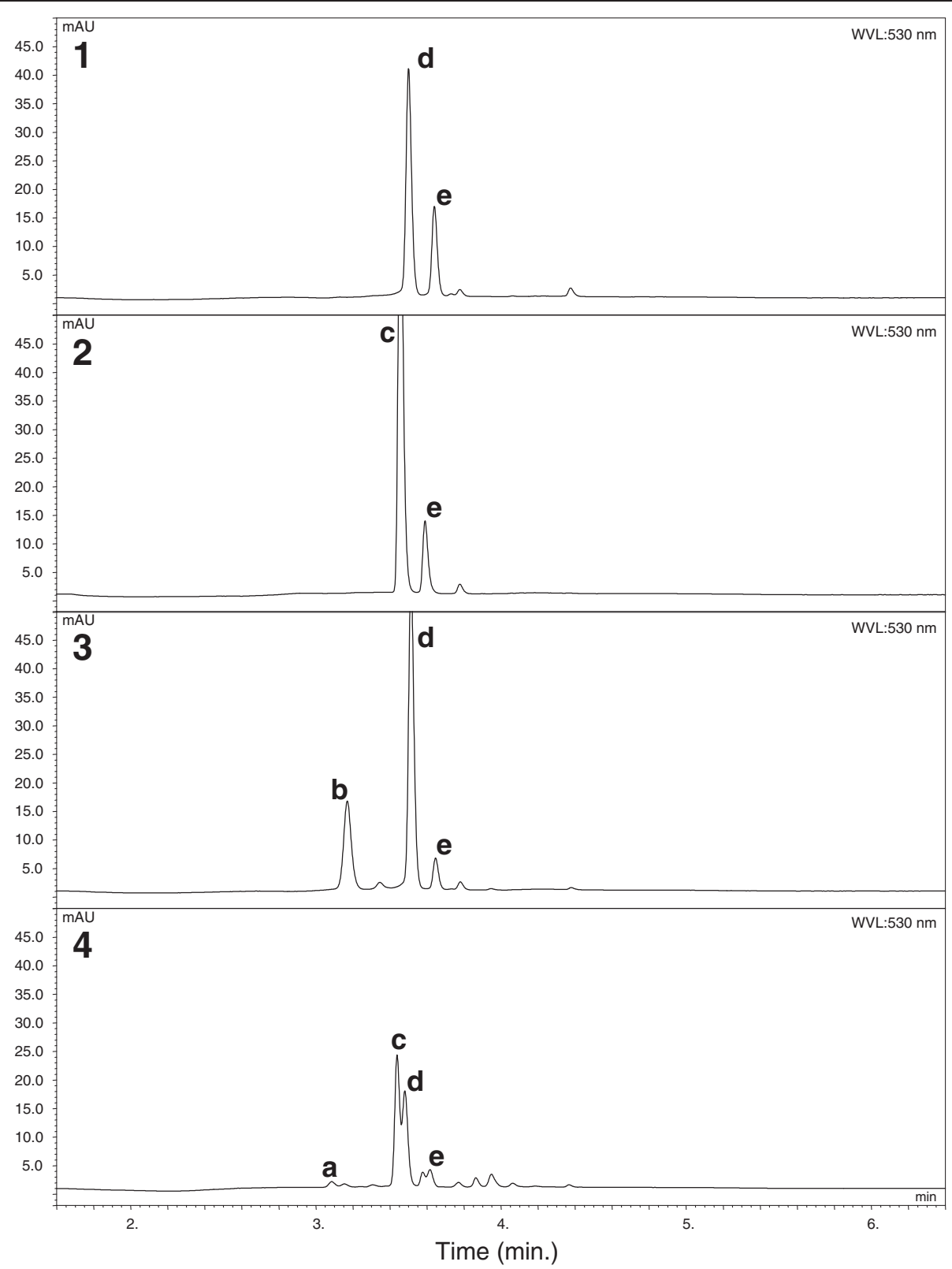

Figure 4 Anthocyanin profiles of red petals of genotypes segregating for this phenotype. Analysis of anthocyanins in red Actinidia petals by Ultra High Performance Liquid Chromatography (UHPLC)(WVL $530 \mathrm{~nm}$ ) identified four patterns that commonly occurred. Traces 1 to 4 show the anthocyanins present, and their concentrations, in the sample as: a - delphinidin 3-O-(xylosyl)galactoside; b - delphinidin 3-O-galactoside; c - cyanidin 3-O-(xylosyl)galactoside; d - cyanidin 3-O-galactoside; e - cyanidin 3-O-glucoside. Trace 1 is dominated by cyanidin 3-Ogalactoside/cyanidin 3-O-glucoside. Cyanidin 3-O-(xylosyl)galactoside is the most plentiful anthocyanin present in trace 2, while trace 3 shows a significant concentration of delphinidin 3-O-galactoside together with cyanidin 3-O-galactoside/cyanidin 3-O-glucoside. Trace 4 shows a pattern of multiple anthocyanins with delphinidin 3-O-(xylosyl)galactoside, delphinidin 3-O-galactoside, cyanidin 3-O-(xylosyl)galactoside, cyanidin 3-O-galactoside and cyanidin 3-O-glucoside all present.

Identification of candidate anthocyanin regulatory factors Previous research has shown the key role of the MYB/bHLH/WDR complex in regulation of the flavonoid pathway, and, in particular a subgroup of R2R3-MYB characterized by the presence of the bHLH interacting signature ([DE] $\left.\mathrm{Lx}_{2}[\mathrm{RK}] \mathrm{x}_{3} \mathrm{Lx}_{6} \mathrm{Lx}_{3} \mathrm{R}\right)$ in the R2R3 domain, and a C-terminus KPRPR[S/T]F motif typical of anthocyanin regulators [27,28]. From an extensive EST database from different species and tissues of Actinidia [26], two R2R3-MYBs were identified, MYB10 and MYB110a, with both these motifs [29]. Further sequencing of kiwifruit genomic sequences led to the identification of a third R2R3-MYB (termed $M Y B 110 b$ ), almost identical in the coding sequence region to $M Y B 110 a$, but 
which proved, through expression studies, to be a different gene and not an allelic variation of MYB110a, and assigned to a different locus by genetic mapping.

In a phylogenetic tree based on the alignment of the deduced amino acid sequences, the three MYB genes: $M Y B 10, M Y B 110 a, M Y B 110 b$, were grouped with the other R2R3-MYB genes shown to be involved in the anthocyanin regulatory process in several plant species (Figures 5a, 5b).

\section{Gel and capillary array electrophoresis of two MYB110} candidate genes in families segregating for petal colour The sequence of each of the MYB110 genes in this study was examined and PCR primer pairs were designed to create microsatellite markers that would identify each gene. MYB110a was represented by marker Ke923 and MYB110b by marker Ke701. The PCR products amplified by the markers of both the MYB110 candidate genes were examined by gel electrophoresis with a sample set of both red and white-petalled progeny from the interspecific backcross. Both markers were seen to be polymorphic, with particular bands segregating with red petal colour.

A single band for MYB110a was amplified in white petal types using Ke923 primers, while a second band clearly amplified in all red petal samples (Figure 6a). Capillary array electrophoresis with these primers revealed products of two sizes amplified in the available parents of the crosses made to produce the $F_{2}$ backcross families (Table 2). The red-petalled A. eriantha or A. eriantha $\mathrm{x}$ $A$. chinensis var. chinensis parents were found to carry alleles corresponding to PCR products of sizes 209 bases (allele 209), and 228 bases (allele 228), and the presence of allele 209 conferred the red phenotype for petal colour. The male parent, $A$. chinensis CK15_02, of the $\mathrm{F}_{2}$ population was homozygous and carried allele 228. This simple allelic pattern in the parents gave rise to redpetalled progeny with alleles 209 and 228, and whitepetalled progeny that were homozygous for allele 228.

Gel electrophoresis with the primer pair representing MYB110b demonstrated that white petal genotypes gave a single band while two bands, with a third minor band, were present in all red petal samples (Figure 6b). With capillary array electrophoresis, a total of ten alleles produced amplification products in the available parents of the families (Table 2). Of the ten alleles shown in parental genotypes, only six were recorded in the progeny of the four families. Of these six alleles, two were found to segregate with red colour, alleles 359 and 361 (Table 2). Family 1 carried allele 361 while families 2, 3 and 4 all carried allele 359 .

\section{Mapping of MYB110a (Ke923), MYB110b (Ke701) and MYB10 (Ke922)}

Three sets of primers were used to amplify markers within MYB10, MYB110a and MYB110b gene regions. Marker Ke922, designed for the MYB10 gene, amplified a female informative marker $[\mathrm{a}(281) \mathrm{b}(275)$ x a(281)a (281)] in a diploid A. chinensis var. chinensis mapping population (see Fraser et al. 2009 for mapping details [30]). Inclusion of this marker into a dataset of microsatellite markers from this population, and using JoinMap 3.0 to group and order markers, linked the gene to linkage group 20. Marker Ke701, designed for the MYB110b gene, amplified a fully informative marker $(\mathrm{a}(413) \mathrm{b}(415) \mathrm{x}$ $\mathrm{c}(426) \mathrm{d}(427))$ in the diploid A. chinensis var. chinensis mapping population and genetic mapping linked this gene to linkage group 10. Marker Ke923, designed for the MYB110a gene, amplified a non-polymorphic marker (aaxaa) in the diploid A. chinensis mapping family, so the position of this gene within the genome was unable to be determined by genetic mapping in A. chinensis. However, in the (A. chinensis var. chinensis $\times$ A. eriantha) $\mathrm{x}$ A. chinensis var. chinensis hybrids, marker Ke701 was fully informative and Ke923 was female informative and the two markers were tightly linked together and also tightly linked with the red petal phenotype (Table 2).

\section{MYB110a expression correlates with anthocyanin synthesis in flower petals}

Cyanidin 3-O-galactoside is an abundant anthocyanin in red petals and is required for the formation of cyanidin 3-O-xylogalactose (Figure 3). F3GT1 is an anthocyanin 3-O-galactosyltransferase previously described in fruit of $A$. chinensis that glycosylates cyanidin 3-O-galactoside in vitro [31]. By quantitative PCR (qPCR) we analysed the level of transcript of F3GT1 in four different genotypes, of which two (21-05-08a and 21-05-11f) had white petals, and two (21-08-12d and 21-05-10h) had bright red petals, at two different stages of flower development: calyx split and full bloom.

Transcript levels of F3GT1 were high in 21-08-12d and 21-05-10h during flower development, but barely detectable in 21-05-08a and 21-05-11f (Figure 7). This result correlates with the degree of pigmentation of the petals. In the genotypes with red petals, the level of transcript of F3GT1 was higher at the calyx split stage of development and decreased as the flower developed to full bloom. In the white flowered genotypes, the level of transcript was barely detectable at either stage tested. Transcript levels of both $M Y B 110 a$ and MYB10 were higher at the early stage of calyx split in the two red petal genotypes, and both were seen to decline at the later stage of full bloom (Figure 7). $M Y B 110 a$ showed a high level of transcript in the red petals, while $M Y B 10$ was expressed, but at a much lower level. cDNA sequence analysis of the expressed transcript confirmed the identification as MYB110a. Primers specific for $M Y B 110 b$ did not amplify any transcript, indicating that $M Y B 110 b$ is not expressed in petals.

In the white petals the level of $M Y B 110 a$ transcript was barely detectable at any stage. $M Y B 110 a$ expression 


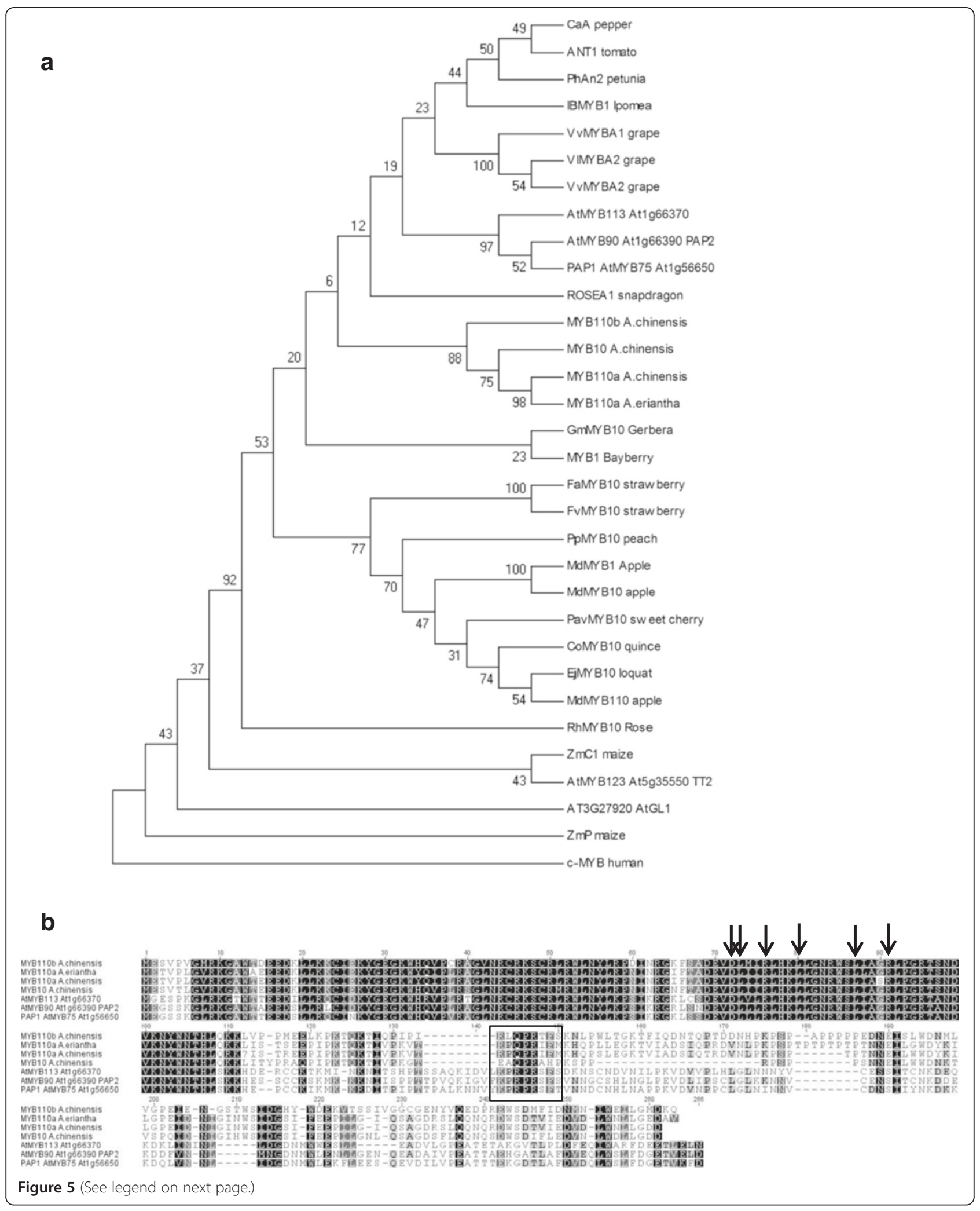


(See figure on previous page.)

Figure 5 Phylogeny of $A$. chinensis and $A$. eriantha MYB genes evaluated from protein sequence. (a). Phylogeny of MYB proteins implicated in anthocyanin regulation in various genera, and the positions assumed by the MYB10 and MYB110 of Actinidia chinensis var. chinensis and A. eriantha. Phylogenetic and molecular evolutionary analysis was conducted using MEGA version 4.0.2 [38] (using minimum evolution phylogeny test and 1000 bootstrap replicates). GenBank numbers; RhMYB10 (ABX79949), ZmP (AF292540), ZmC1 (Y15219), IbMYB1 (AB576767), GmMYB10 (ACM62751), ROSEA1 (DQ275529), MYB1 bayberry (GQ340767), VvMYBA2 (ABL14065), VIMYBA2 (BAC07540), VvMYBA1 (AB242302), ANT1 (AY348870), PhAn2 (EF423868), CaA (AJ608992), FvMYB10 (ABX79948), FaMYB10 (ABX79947), PpMYB10 (ABX79945), PavMYB10 (ABX71493), MdMYB110 (EB710109), EjMYB10 (ABX71484), CoMYB10 (ABX71483), MdMYB10 (ACQ45201), MdMYB1 (DQ886414) and c-MYB (AAB49039). (b). Protein sequence data from which phylogenetic relationships of MYB genes were evaluated. Sequences were aligned using Clustal W (opening = 15, extension-0.3) in Vector NT19.0. Arrows indicate the amino acid signature motif ([DE]Lx $\left.[R K] x_{3} L x_{6} L x_{3} R\right)$ in the R2R3 domain, which allows interaction with a bHLH partner, and the box shows the C-terminus motif typical of anthocyanin-related regulators.

appeared to be strongly correlated with F3GT1 expression, being present in the same tissue and showing the same pattern of expression. Both were highly expressed at calyx split, with expression declining at full bloom. This strong link between the transcript level of $M Y B 110 a$ and F3GT1 suggests the involvement of $M Y B 110 a$ in the regulation of the transcription of F3GT1, and of the anthocyanin biosynthesis pathway in the petals of $A$. eriantha, and of the studied population.

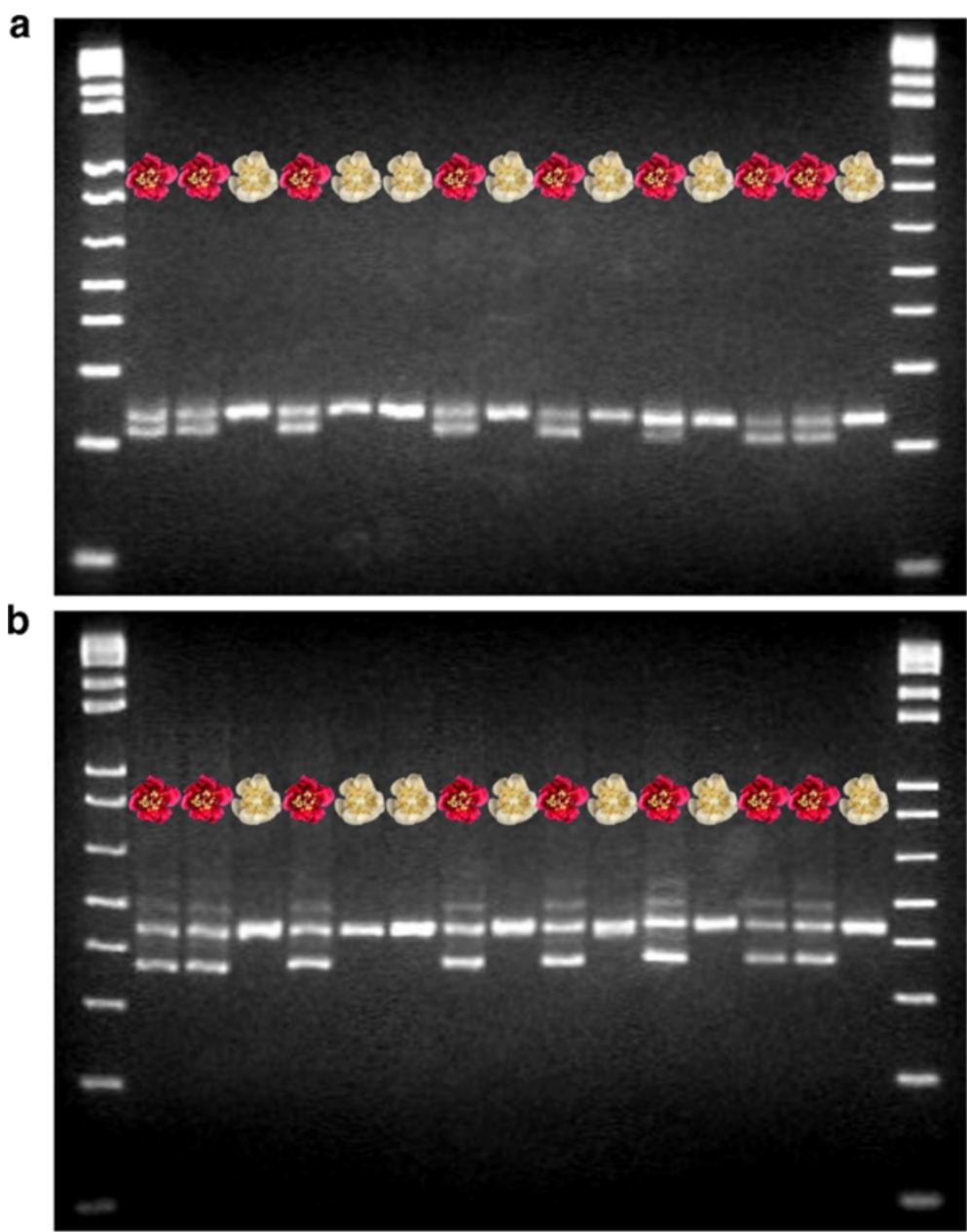

Figure 6 Gel electrophoresis banding patterns identify red-petal progeny. Progeny of four interspecific hybrid families between (Actinidia chinensis var. chinensis $\times$ Actinidia eriantha) $\times$ Actinidia chinensis var. chinensis had either red or white-petalled flowers. Gel electrophoresis of the PCR products amplified with primers to the MYB110 genes showed that the genotypes could be distinguished on the basis of their banding patterns. With MYB110a, (primer pair Ke923) genotypes with red petal colour all had two clear bands, while genotypes with white petal colour had a single band (Figure 6a). Primer pair Ke701, specific to MYB110b, also segregated with red petal colour (Figure 6b). In this instance the red petal phenotype had three bands while the white petal genotype had one band. Both gels were run through a $2.75 \%$ agarose gel with a $1 \mathrm{~Kb}+$ size ladder. 
Table 2 Allelic composition of the parents and progeny of the $F_{2}$ hybrid backcross

\begin{tabular}{|c|c|c|c|c|c|c|c|c|c|c|c|c|c|c|c|}
\hline Progeny sample & G & Fam & Petal colour & Ke923 & 110a) alleles & Ke701 & 110b) all & & & & & & & & \\
\hline & & & & 1 & 2 & 1 & 2 & 3 & 4 & 5 & 6 & 7 & 8 & 9 & 10 \\
\hline $21-05-08 g$ & $m$ & 1 & red & 209 & 228 & & 361 & & & & & & 426 & & \\
\hline 21-05-08j & $\mathrm{m}$ & 1 & red & 209 & 228 & & 361 & & & & & & 426 & & \\
\hline $21-07-09 c$ & $f$ & 1 & red & 209 & 228 & & 361 & & & & & & 426 & & \\
\hline 21-06-10h & $f$ & 1 & red & 209 & 228 & & 361 & & & & & & & 427 & \\
\hline 21-07-09f & $\mathrm{m}$ & 1 & red & 209 & 228 & & 361 & & & & & & & 427 & \\
\hline 21-07-09g & $f$ & 1 & red & 209 & 228 & & 361 & & & & & & & 427 & \\
\hline $21-07-09 b$ & $f$ & 1 & white & & 228 & & & & & & 410 & & 426 & & \\
\hline $21-07-12 e$ & $\mathrm{~m}$ & 1 & white & & 228 & & & & & & 410 & & 426 & & \\
\hline 21-08-08h & $f$ & 1 & white & & 228 & & & & & & 410 & & 426 & & \\
\hline 21-07-09a & $\mathrm{m}$ & 1 & white & & 228 & & & & & & 410 & & & 427 & \\
\hline $21-07-12 b$ & $f$ & 1 & white & & 228 & & & & & & 410 & & & 427 & \\
\hline $21-08-08 b$ & $f$ & 1 & white & & 228 & & & & & & 410 & & & 427 & \\
\hline 21-05-09f & $\mathrm{m}$ & 2 & red & 209 & 228 & 359 & & & & & & & 426 & & \\
\hline $21-07-13 d$ & $\mathrm{~m}$ & 2 & red & 209 & 228 & 359 & & & & & & & 426 & & \\
\hline 21-06-11i & $f$ & 2 & red & 209 & 228 & 359 & & & & & & & & 427 & \\
\hline 21-07-13e & $f$ & 2 & red & 209 & 228 & 359 & & & & & & & & 427 & \\
\hline 21-08-11a & $\mathrm{m}$ & 2 & red & 209 & 228 & 359 & & & & & & & & 427 & \\
\hline $21-05-09 b$ & $\mathrm{~m}$ & 2 & white & & 228 & & & & & & & & 426 & & 432 \\
\hline 21-05-09e & $f$ & 2 & white & & 228 & & & & & & & & 426 & & 432 \\
\hline 21-05-09h & $\mathrm{m}$ & 2 & white & & 228 & & & & & & & & 426 & & 432 \\
\hline $21-05-09 g$ & $f$ & 2 & white & & 228 & & & & & & & & & 427 & 432 \\
\hline $21-07-13 g$ & $f$ & 2 & white & & 228 & & & & & & & & & 427 & 432 \\
\hline $21-07-14 \mathrm{~g}$ & $\mathrm{~m}$ & 2 & white & & 228 & & & & & & & & & 427 & 432 \\
\hline $21-05-12 i$ & $f$ & 3 & red & 209 & 228 & 359 & & & & & & & 426 & & \\
\hline $21-07-11 c$ & $\mathrm{~m}$ & 3 & red & 209 & 228 & 359 & & & & & & & 426 & & \\
\hline 21-09-10c & $f$ & 3 & red & 209 & 228 & 359 & & & & & & & 426 & & \\
\hline $21-05-12 a$ & $f$ & 3 & red & 209 & 228 & 359 & & & & & & & & 427 & \\
\hline $21-05-12 \mathrm{~g}$ & $f$ & 3 & red & 209 & 228 & 359 & & & & & & & & 427 & \\
\hline 21-07-11b & $\mathrm{m}$ & 3 & red & 209 & 228 & 359 & & & & & & & & 427 & \\
\hline 21-06-13a & $\mathrm{m}$ & 3 & white & & 228 & & & & & & & & 426 & & \\
\hline 21-08-09h & $f$ & 3 & white & & 228 & & & & & & & & & 427 & \\
\hline 21-06-08a & $f$ & 4 & red & 209 & 228 & 359 & & & & & & & 426 & & \\
\hline $21-06-12 b$ & $\mathrm{~m}$ & 4 & red & 209 & 228 & 359 & & & & & & & 426 & & \\
\hline 21-07-08h & $f$ & 4 & red & 209 & 228 & 359 & & & & & & & 426 & & \\
\hline 21-07-10b & $\mathrm{m}$ & 4 & red & 209 & 228 & 359 & & & & & & & 426 & & \\
\hline 21-09-11f & $\mathrm{m}$ & 4 & red & 209 & 228 & 359 & & & & & & & 426 & & \\
\hline 21-06-08h & $f$ & 4 & red & 209 & 228 & 359 & & & & & & & & 427 & \\
\hline 21-07-10h & $f$ & 4 & red & 209 & 228 & 359 & & & & & & & & 427 & \\
\hline 21-09-09a & $f$ & 4 & red & 209 & 228 & 359 & & & & & & & & 427 & \\
\hline 21-09-09d & $\mathrm{m}$ & 4 & red & 209 & 228 & 359 & & & & & & & & 427 & \\
\hline 21-07-08a & $\mathrm{m}$ & 4 & white & & 228 & & & & & & & & 426 & & \\
\hline $21-07-08 b$ & $f$ & 4 & white & & 228 & & & & & & & & 426 & & \\
\hline
\end{tabular}


Table 2 Allelic composition of the parents and progeny of the $\mathbf{F}_{\mathbf{2}}$ hybrid backcross (Continued)

\begin{tabular}{|c|c|c|c|c|c|c|c|c|c|c|c|c|c|}
\hline $21-07-10 e$ & $f$ & 4 & white & & 228 & & & & & & & 426 & \\
\hline 21-07-10f & $\mathrm{m}$ & 4 & white & & 228 & & & & & & & 426 & \\
\hline \multicolumn{14}{|l|}{ Parents } \\
\hline CK01_03 & $f$ & & white & nd & & & & & & 410 & 413 & & \\
\hline CK01F2 & $f$ & & white & & 228 & & & 406 & 408 & & & & \\
\hline CK15_01 & $\mathrm{m}$ & & white & & 228 & & & & & & & 426 & \\
\hline CK15_02 & $\mathrm{m}$ & & white & & 228 & & & & & & & 426 & 427 \\
\hline CK21_01 & f & & white & & 228 & & & & 409 & & & & 432 \\
\hline EA03_01 & $f$ & & red & 209 & & 359 & 361 & & & & & & \\
\hline EA04_03 & $f$ & & red & 209 & 228 & 359 & & & & & & & \\
\hline EA07_R22 & $\mathrm{m}$ & & red & 209 & 228 & nd & & & & & & & \\
\hline Hort16A & f & & white & & 228 & & & 406 & & & & 426 & \\
\hline $11-06-17 c$ & f & 1 & red & 209 & 228 & & 361 & & & 410 & & & \\
\hline $11-06-16 e$ & f & 2 & red & 209 & 228 & 359 & & & & & & & 432 \\
\hline $11-06-15 d$ & f & 3 & red & 209 & 228 & 359 & & & & & & 426 & \\
\hline $11-06-15 c$ & f & 4 & red & 209 & 228 & 359 & & & & & & 426 & \\
\hline
\end{tabular}

Analysis by capillary electrophoresis of PCR products with the MYB110 gene markers showed that both MYB110a and MYB110b carried alleles that segregated with red petal colour. In all the parents available for analysis, and progeny, only two alleles were amplified with MYB110a marker primers. Red-petalled Actinidia genotypes carried alleles represented by PCR products sized 209 (allele 209) and 228 (allele 228), while white-petalled genotypes were homozygous for allele 228. Analysis with MYB110b showed a total of ten alleles amplified in parents. Of these ten alleles, only six were found in the $F_{2}$ backcross progeny. Two of the six alleles, alleles 359 and 361 , segregated with red petal colour. The presence of either allele gave the red phenotype.

\section{MYB110a regulates F3GT1 expression and anthocyanin biosynthesis}

To assess the ability of MYB110a to regulate gene expression of the anthocyanin pathway in Actinidia flower petals, it was determined if MYB110a was able to trans-

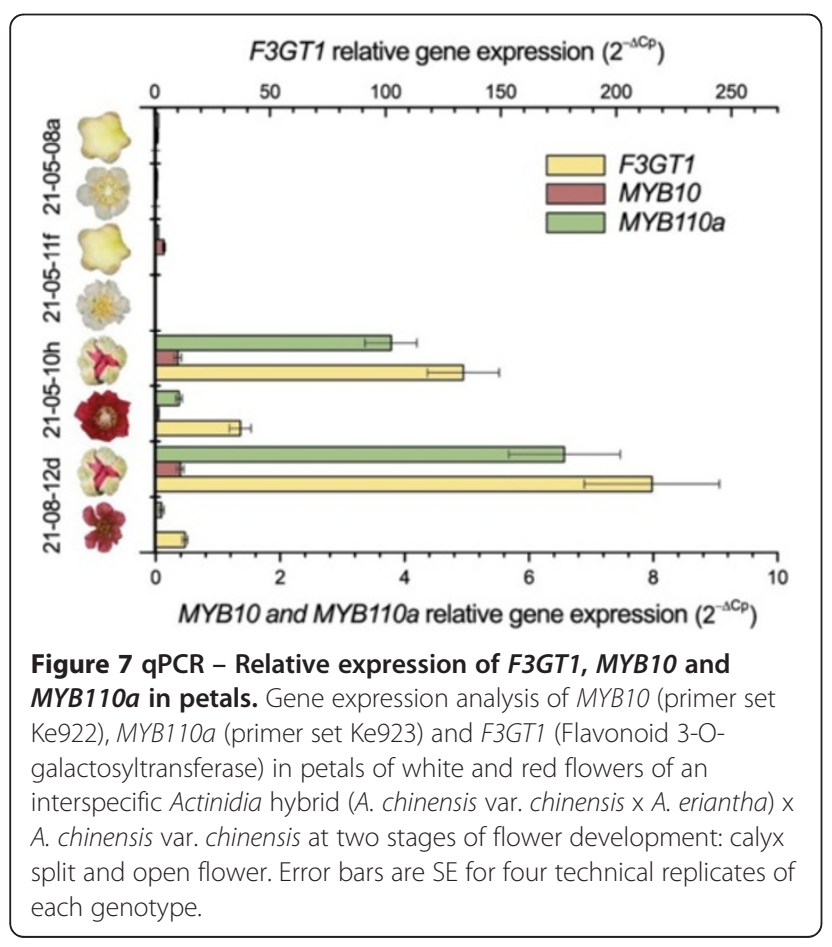

activate the Actinidia F3GT1 promoter in a transient luciferase assay performed in Nicotiana benthamiana leaves. The luciferase assay showed that the F3GT1 promoter was strongly activated by MYB110a (Figure 8). Co-transformation of MYB110a with the Arabidopsis bHLH TT8 slightly increased luciferase activity. A similar result was obtained when the Arabidopsis R2R3MYB AtMYB75 (PAP1) was used, while no activation occurred when the bHLH AtTT8 alone was tested (Figure 8). When an Arabidopsis promoter $A t D F R$ [32], fused to luciferase was used, MYB110a showed strong activation with and without AtTT8, while AtMYB75 was significantly stimulated by the presence of AtTT8. This suggests that MYB110a was either able to recruit endogenous tobacco bHLH and WDR and form the MBW complex to induce promoter activation, or other co-factors are involved.

MYB110a was transiently over-expressed in the petals of a white-petalled individual (21-05-09e) of the $F_{2}$ backcross population. Agrobacterium strain GV3101, carrying a plasmid (pHEX2) harbouring 35S::MYB110a, was syringed into a small cut in the petal edge. In the area surrounding the site of infiltration, over-expression of $M Y B 110$ a complemented the white phenotype and restored the ability to synthesise anthocyanin in petals (Figures 9a, 9b). Microscopy of a petal demonstrating transient expression (Figure 9c) showed that anthocyanin pigmentation varied in concentration within the pigmented area (Figure 9d) and was confined within individual 


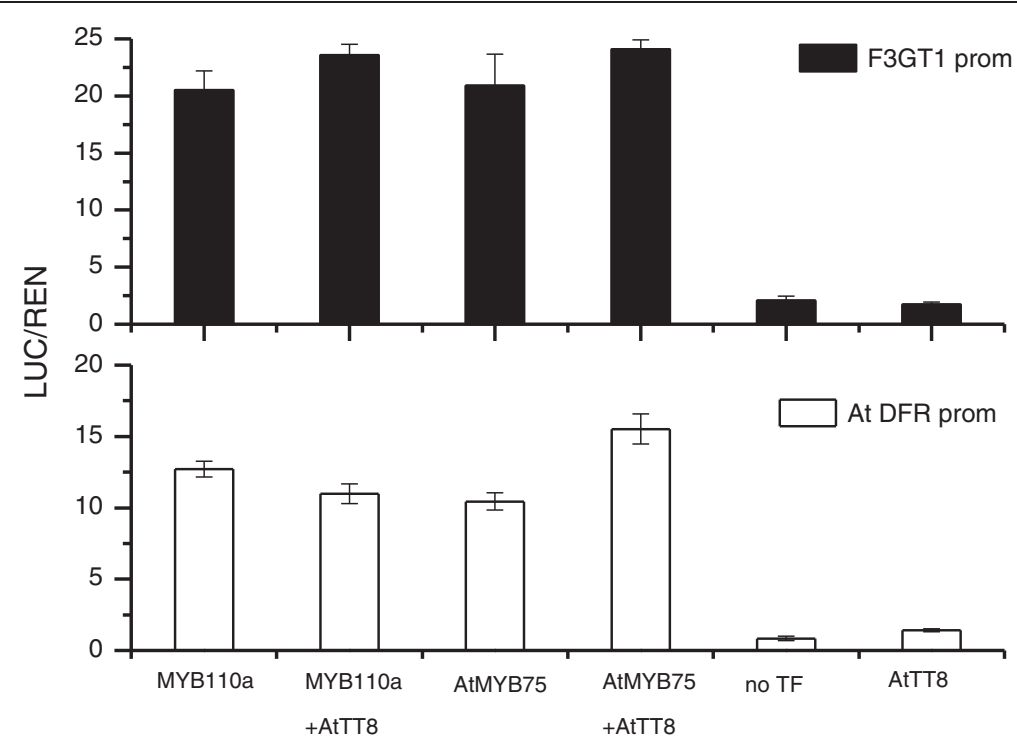

Figure 8 Trans-activation of the F3GT1 and AtDFR promoters by MYB110a and AtMYB75 in a dual luciferase transient assay. Leaves of Nicotiana benthamiana were infiltrated with F3GT1 promoter-LUC or AtDFR promoter-LUC fusions on their own or Co-infiltrated with 35S::MYB110a, 35S::AtMYB75 or with 355::AtTT8. Luminescence of LUC and REN was measured 3 d later and expressed as a ratio of LUC to REN. Data are presented as means $( \pm$ SE) of four biological replicates of each transgene combination.

cells (Figure 9e). Transient over-expression of green fluorescent protein (GFP) in petals showed that Agrobacterium alone does not elicit an anthocyanin response in white petals (Figure 9f, 9g, 9h). The luciferase assay and the complementation of white petal phenotype by overexpression of MYB110a demonstrate the ability of this transcription factor to activate and regulate the anthocyanin biosynthetic pathway in petals of the Actinidia hybrid population.

\section{Discussion}

Previous studies have concentrated on the characterization of red colour in the fruit of Actinidia species [33,34]. In those studies three cyanidin-based and two delphinidinbased anthocyanins were identified in fruit of six Actinidia species. While the cyanidin compounds were found in all the species examined, only two taxa, A. melanandra and A. arguta var. purpurea, contained the delphinidin-based compounds: delphinidin 3-O-(xylosyl)galactoside and delphinidin 3-O-galactoside. They were not found in either A. chinensis or $A$. eriantha. In our study we found that both delphinidin compounds were present in the petals in a proportion of the progeny genotypes within three of the four families resulting from a backcross between A. eriantha and A. chinensis var. chinensis. Cyanidin and delphinidin are produced through different branches of the anthocyanin pathways, from the action of either the flavonoid 3'-hydroxylase (F3'H) or flavonoid 3'5'-hydroxylase $\left(F 3^{\prime} 5^{\prime} H\right)$, so our results suggest that both pathways are activated in the flowers of the Actinidia backcross population studied here [34].
Anthocyanin colour perception in plant tissues is affected by the ability of the anthocyanins to undergo changes in chemical form resulting from their interactions with metal ions, other anthocyanin molecules (self-association), or other unrelated compounds (co-pigmentation). These associations may cause a change in hue, or an increase in colour intensity [35,36]. A wide range of potential co-pigment compounds have been reported, but by far the most common are the colourless flavonol and flavone types of flavonoids. Flavonols were found in the petal samples examined here, with three of the four flavonols identified, quercetin-rutinoside, quercetin-glucoside and kaempferol-glucoside, being present in all samples. While kaempferol-rutinoside was present in 60 of the 134 samples, it was only found in small concentrations, and its presence or absence did not correlate with the perceived hue of the petals. Neither was the presence or absence of delphinidin compounds aligned with perceived hue. This can be seen from the two genotypes 21-07-11a and 21-09-13d, where delphinidin was not present in either genotype but petals of 21-09-13d appeared to have a blue tinge (Figure 2). Therefore we conclude that the hue variation observed in the different genotypes cannot be explained by the combinations of anthocyanin or flavonol types recorded. The differences in depth of colour seen in the petals corresponded to the total anthocyanin concentration.

This study indicates that anthocyanin and flavonol biosynthesis in Actinidia is a complex and highly regulated process. The flavonol kaempferol and the anthocyanin pelargonidin share the same dihydrokaempferol (DHK) 


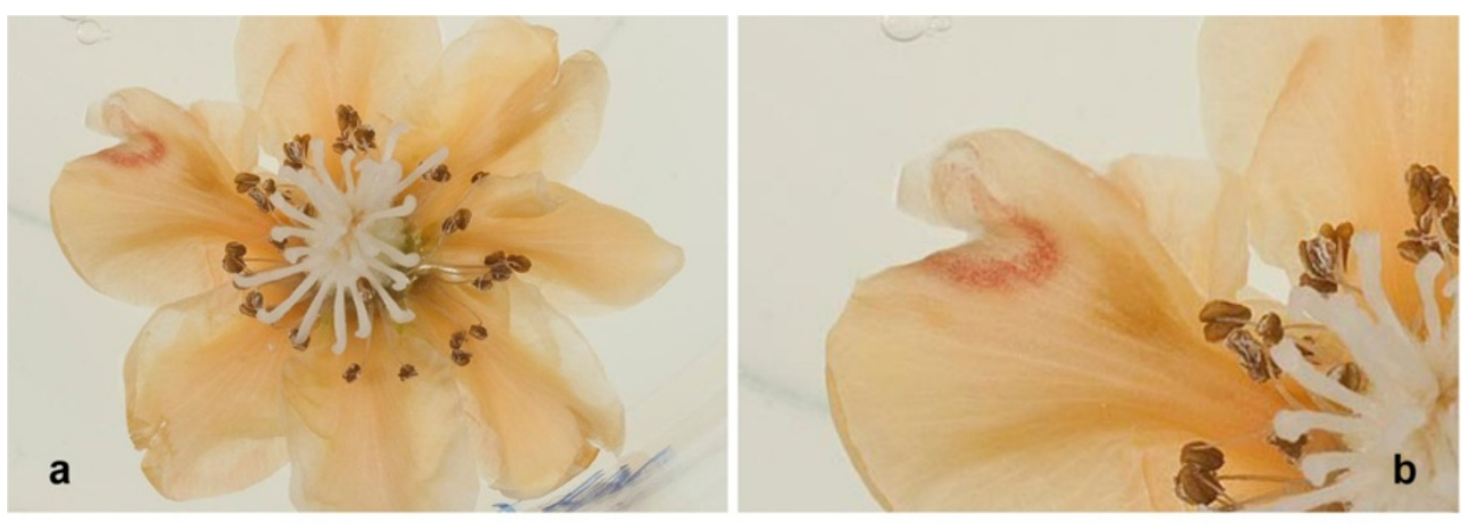

Flower transfected with 35S:MYB110a at the petal edge
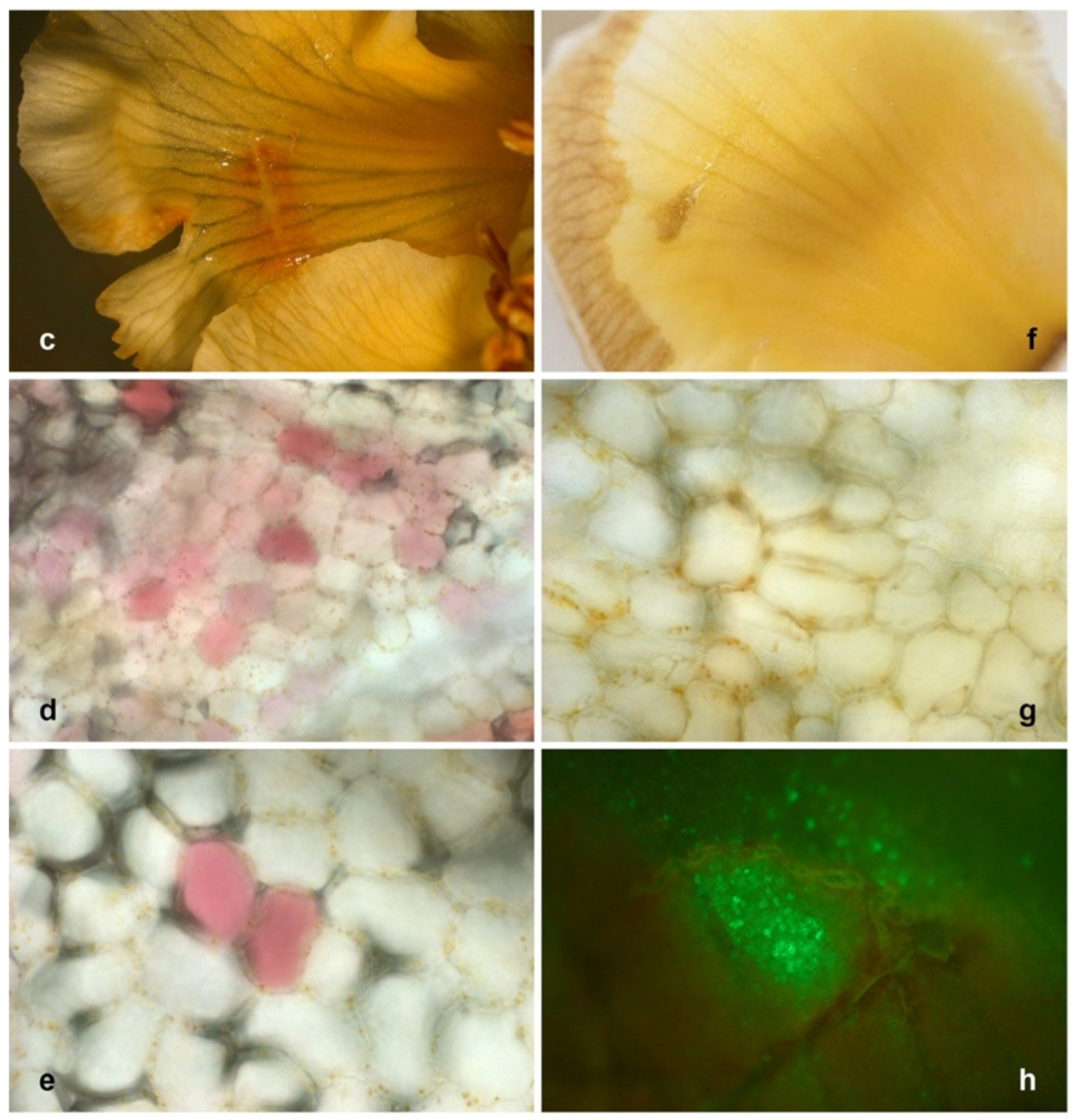

35S::AcMyb110

$35 S:: G F P$

Figure 9 (See legend on next page.) 


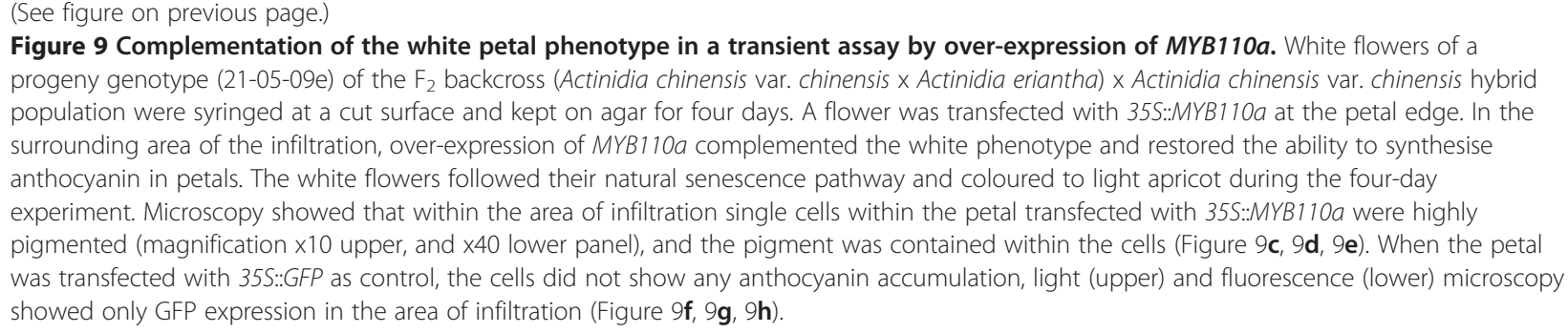

precursor. However, while kaempferol was present in the petals, the DHK was not apparently utilised by dihydroflavonol 4-reductase (DFR) and anthocyanidin synthase (ANS) to produce pelargonidin, as this compound was not detected. Rather, the biosynthetic route to anthocyanins proceeded by the action of F3' $\mathrm{H}$ and F3'5' $\mathrm{H}$ on the precursors prior to their conversion by DFR and ANS to cyanidin (3'4'-hydroxylation) and delphinidin (3'4'5'hydroxylation). The presence of F3'5'H activity, however, did not result in the production of the flavonol myricetin, which also has $3^{\prime} 4^{\prime} 5^{\prime}$-hydroxylation. The apparent channelling of substrate into flavonols and anthocyanins with varying patterns of hydroxylation, despite the presence of the $\mathrm{F}^{\prime} \mathrm{H}$ and $\mathrm{F}^{\prime} 5^{\prime} \mathrm{H}$, has also been observed in other species [2]. It could reflect different developmental timings for the production of flavonols and anthocyanins, metabolic channelling within enzyme complexes, or particular substrate specificity of biosynthetic enzymes such as DFR. The DFR of genera such as Petunia and Cymbidium have been shown to have only weak activity with DHK, resulting in the near absence of pelargonidin-based anthocyanins in these genera [37].

In general, flavonoids with a free hydroxyl group at the $\mathrm{C}-3$ position of the heterocyclic ring are unstable under physiological conditions, and are therefore typically found as their glycosylated forms. Unlike the case in many plants, differential glycosylation occurs at the C-3 position for flavonols and anthocyanins of Actinidia. The flavonols identified were glucosides and rutinosides (resulting from addition of rhamnose to the glucose), while the main anthocyanins were galactosides and xylosyl-galactosides. Only low concentrations of anthocyanin-glucosides were found, and anthocyaninrutinosides were not detected.

Three R2R3-MYB genes have been identified for Actinidia that show the conserved amino acid sequences of activators of anthocyanin biosynthesis $M Y B 10, M Y B 110 a$ and MYB110b. MYB110a is thought likely to be the major gene defining petal colour as it was expressed at high levels in red but not white petals, and $M Y B 110 b$ expression was not detected at all. Expression for $M Y B 110 a$ (and to a lesser extent, $M Y B 10)$ was highest before full bloom, and declined as the flower expanded. This pattern occurs with anthocyanin-related MYBs controlling other floral phenotypes, eg. petunia [11]. The white-petalled phenotype of flowers was complemented through introduction of $M Y B 110 a$, confirming its identity as an anthocyanin regulator. Furthermore, specific gene markers were obtained that linked different alleles of MYB110a to the presence of white versus red petals. The results show that $M Y B 110 a$ is the agent of specificity of anthocyanin expression in the flower petals while $M Y B 110 b$ is probably a tightly linked, but not expressed, relative of $M Y B 110 a$. Fruit flesh and flower ovary and filament colour must be under the control of other R2R3-MYB gene family members, or alleles, as these did not segregate with MYB110a. Future work will concentrate on identifying fruit-specific R2R3-MYB markers for use in marker assisted breeding.

In transient assays, adding exogenous bHLH (AtTT8) had surprisingly little effect on the activation of the F3GT1 and AtDFR promoters by MYB110a. However, it is unlikely that MYB110a functions without a bHLH. Instead MYB110a may be more efficient at interacting with tobacco bHLHs already present in the transiently expressing cell, and this could be determined using hairpins to tobacco endogenous bHLH genes. Although the bHLH is thought to be the main component that links with the different WDR, R2R3- and R3-MYB proteins in the MBW regulatory complex, variation in bHLH expression is unlikely to provide for tissue-specific anthocyanin production. The results in our study of Actinidia support the proposal that variation in R2R3-MYB activator function and expression is the key determinant of spatial and temporal patterning of anthocyanin production in most plant species $[15,16]$.

\section{Conclusions}

We have shown that the red petal phenotype in the interspecific Actinidia population is the result of a mixture of anthocyanins, with Cy-gal present in the greatest concentration, and with Cy-xylgal and Cy-glu also present in significant amounts, and in various combinations, in individual genotypes. Other anthocyanins were present in minor quantities in different genotypes. Delphinidin was recorded as both delphinidin 3-O-(xylosyl) 
galactoside (dp-xylgal) and delphinidin 3-O-galactoside (dp-gal) and could be considered in parent choice for breeding populations for colour variation in fruit. Red or white petal colour in $A$. chinensis x $A$. eriantha hybrids is determined by MYB110a.

\section{Methods}

\section{Plant material and DNA extraction}

Four interspecific backcross families between A. chinensis var. chinensis and $A$. eriantha were created as detailed in Additional file 1. The population was grown at the Plant \& Food Research Te Puke Research Centre, Bay of Plenty, New Zealand. At budbreak, leaf tissue was taken from each genotype, held at $4^{\circ} \mathrm{C}$ for $24 \mathrm{~h}$, and stored at $-80^{\circ} \mathrm{C}$ until required. DNA was extracted from leaves about $1 \mathrm{~cm}$ in length from all progeny, and as many of the parents of the crosses as were available. The sample was ground to powder in liquid nitrogen before being processed through a DNeasy Plant Mini Kit (Qiagen ${ }^{\mathrm{Tu}}$ ) according to the manufacturer's instructions. The final eluate was $200 \mu \mathrm{l}$ in volume. Five $\mu \mathrm{l}$ of a one in ten dilution of this eluate was used in each PCR reaction.

With the goal of a usable genetic marker in Actinidia, the female and male parents of an $\mathrm{F}_{1}$ Actinidia genetic mapping population [30] were also included. The female parent of the mapping population had white flowers and red colour in the fruit, while the male parent carried white flowers and no evidence of red flesh in male line siblings. The male parent of the mapping population was also the male parent of all four families in this study.

\section{Phenotyping of floral structures and fruit}

In October 2009, flowers from 275 backcross hybrid seedlings were scored for the presence of red colour in their petals. Two flower buds from each of 101 female seedlings were dissected and scored for the presence of red colour in the pericarp tissue of the ovary. Flowers from three of the four female $F_{1}$ hybrid parents of the backcross population were also scored for these traits (the fourth parent, 11-06-15c, produced no flowers in 2009). Flowers of the male $A$. chinensis parent involved in the backcross were scored for petal colour. The sex of each seedling was recorded, based on the morphology of the flowers. In 2010, ripe fruit from 88 female vines were screened for the presence of red colour in the fruit flesh.

\section{Candidate gene identification. Microsatellite identification and primer design}

The candidate genes, MYBs, were identified in the Plant \& Food Research Actinidia expressed sequence tag (EST) database (available in GenBank, FG527909, FG403522).
Exon and intron sequence data were examined to identify dinucleotide microsatellites that would be suitable for marker identification of the MYB genes. Primer pair sequences were chosen which gave a theoretical PCR product size between 200 and $250 \mathrm{bp}$, with an annealing temperature between $58^{\circ} \mathrm{C}$ and $60^{\circ} \mathrm{C}$, and with a GC content of approximately $50 \%$. As the $M Y B 110 a$ gene closely resembled the neighbouring $M Y B 110 b$ gene in 5 ' sequence, the primers were designed to amplify a sequence at the 3' end of the MYB110a gene. Primer 3 software was used to identify and reduce the incidence of hairpins and primer dimers. One of the primers of the pair was located in an intron sequence while the other was designed within the exon to facilitate marker specificity.

\section{Polymerase chain reaction, electrophoresis, and analysis}

The primer pairs were screened for PCR amplification in available parents and progeny in samples of the four families of the interspecific cross. A reaction mix of $15 \mu \mathrm{l}$ containing $1 \times$ PCR buffer $(20 \mathrm{mM}$ Tris- $\mathrm{HCl}, 50 \mathrm{mM}$ $\mathrm{KCl}$ ), $\mathrm{MgCl}_{2} 5 \mathrm{mM}$ (the buffer and $\mathrm{MgCl}_{2}$ were those supplied with the polymerase), $0.2 \mathrm{mM}$ each of dNTPs, 4.5 pmol of each primer, and 1.25 units of Platinum Taq polymerase (Invitrogen), was prepared for each DNA sample. About $12.5 \mathrm{ng}$ of genomic DNA was added in $5 \mu \mathrm{l}$ to bring the total PCR volume to $20 \mu \mathrm{l}$. PCRs were performed in a Techne ${ }^{\mathrm{TM}}$ TC-412 thermal cycler with a single cycle of $94^{\circ} \mathrm{C}$ for 3 min preceding 35 cycles of denaturing at $94^{\circ} \mathrm{C}$ for $30 \mathrm{sec}$, annealing for $30 \mathrm{sec}$, and elongation at $72^{\circ} \mathrm{C}$ for $1 \mathrm{~min}$. PCR reactions were carried out with primers that were labelled with 6FAM, and prepared for analysis. The allelic content of each genotype was determined by capillary electrophoresis in an ABI Prism ${ }^{\circledR} 3100$ Genetic Analyzer (Filter Set D, ROX $^{\mathrm{sm}}$ size standard), and analyzed with GeneMapper ${ }^{\mathrm{TM}}$ Software Version 3.7 (Applied Biosystems).

\section{Real time qPCR expression analysis}

Petals were collected from individual genotypes, representing both red and white petal types, at late calyx split and full bloom. The samples were immediately frozen in liquid nitrogen in the field, and held at $-80^{\circ} \mathrm{C}$.

RNA was isolated from petals [31] and DNAse-treated (DNA-free:Ambion. http://www.ambion.com/). Reverse transcription was performed using oligo(dT) according to the manufacturer's protocol (Transcriptor: Roche Diagnostics, http://www.roche.com/).

Genes were identified by BLAST with genes of known function in an Actinidia EST database [26]. Phylogeny of $M Y B$ proteins were generated from alignments (using Clustal W) and molecular evolutionary analysis conducted using MEGA version 4.0.2 [38] (using minimum evolution phylogeny test and 1000 bootstrap replicates). 
Gene-specific primers were designed using Vector NTI 9.0.0 (http://www.invitrogen.com) and are summarised in a supplementary table (Additional file 5). Quantitative real-time PCR was carried out using the LightCycler 480 System (Roche Diagnostics), reactions were performed in quadruplicate and a non-template control was included in each run. Thermal cycling conditions were $95^{\circ} \mathrm{C}$ for five min, then 40 cycles of $95^{\circ} \mathrm{C}$ for $10 \mathrm{sec}, 60^{\circ} \mathrm{C}$ for $10 \mathrm{sec}$, and $72^{\circ} \mathrm{C}$ for $15 \mathrm{sec}$, followed by a melting temperature cycle, with constant fluorescence data acquisition from $65^{\circ} \mathrm{C}$ to $95^{\circ} \mathrm{C}$. The data were analysed using the Target/Reference ratio calculated with the LightCycler ${ }^{\circledR} 480$ software 1.5 , enabling a comparison of the level of expression of two different genes normalised to two reference genes: actin and subunit protein phosphatase 2A (PP2A) [31]. To confirm primer specificity, the qPCR product was sequenced (The Allan Wilson Centre: http://www.allanwilsoncentre.ac.nz).

\section{Complementation study by transient expression in petals and dual luciferase assay}

DNA constructs have been created to over-express the MYB/bHLH in tobacco (Nicotiana benthamiana) leaves and Actinidia petals: pHex2-MYB110a, pHex2-AtMYB75, pHex2-AtTT8 and 1Kb promoter sequence of F3GT1 inserted into pGreen 0800-LUC for a dual luciferase assay [39], or the AtDFR promoter fused to LUC as described in Espley et al. [32]. Agrobacterium tumefaciens GV310 (MP90) were transformed with the different constructs. Agrobacterium cultures were resuspended in infiltration buffer prior to infiltration [39]. Flowers from 21-05-09e, a white-flowered genotype, were selected for transient expression studies. A small incision in the petal was required to allow infiltration of the Agrobacterium suspension and after 3 days it was possible to assess the red pigmentation induced by the over-expressed MYB.

Promoter activation, dual luciferase assay, was performed on $N$. benthamiana leaves (four replicates per treatment). The promoter was co-infiltrated with the different transcription factors tested. The F3GT1 promoter sequence was fused to the luciferase reporter gene $L U C$, while in the same construct another luciferase r-gene $R E N$, under the control of a $35 \mathrm{~S}$ promoter, was used as an internal control and as indicator of the extent of the transient expression. Three days after inoculation, firefly luciferase and Renilla luciferase were assayed using the dual luciferase assay reagent (Promega, Madison, WI, USA). Activity is expressed as a ratio of $L U C$ to REN activity [39].

\section{Anthocyanin identity and quantity analyses}

A single bud at the early petal emergence stage of development was collected from each flowering genotype. Each bud was dissected within $1 \mathrm{~h}$ of collection. An inner petal was selected to reduce the possibility that colour may have faded during exposure to light. As anthocyanin production is sensitive to environmental factors, all measurements were made in a single flowering season from plants grown in one area. Anthocyanins were extracted from petals by soaking in a solvent mixture composed of ethanol/water/formic acid (80/20/1). Screw capped vials were prepared containing $1.0 \mathrm{~mL}$ solvent and $50 \mu \mathrm{g}$ of quercetin (internal standard). Weighed portions of petals were added to the vials, which were then capped, frozen at $-20^{\circ} \mathrm{C}$ and transported to Plant \& Food Research, Palmerston North, for analysis.

Ultra High Performance Liquid Chromatography (UHPLC) was used to separate and measure the anthocyanins present in extracts of petal tissue. The UHPLC system used was a Dionex Ultimate ${ }^{\circledR} 3000$ Rapid Separation LC system equipped with a binary pump (HPR-3400RS), autosampler (WPS-3000RS), column compartment (TCC-3000RS), and a diode array detector (DAD-3000RS). The analytical column employed was a Zorbax SB-C18 HD 2.1 x 150 mm, $1.8 \mu \mathrm{m}$ (Agilent Technologies, Santa Clara, United States) maintained at $45^{\circ} \mathrm{C}$. A binary solvent programme was used with Solvent A (formic acid/MQ water 5:95) and Solvent B (acetonitrile) at a flow of $450 \mu \mathrm{L} / \mathrm{min}$. The initial solvent composition was 5\%A 95\%B until 0.5 minutes, then changed to $70 \% \mathrm{~A} 30 \% \mathrm{~B}$ at $3.5 \mathrm{~min}, 60 \% \mathrm{~A} 40 \% \mathrm{~B}$ at $9.0 \mathrm{~min}$, and $20 \% \mathrm{~A} 80 \% \mathrm{~B}$ at $9.5 \mathrm{~min}$. After a 2 -min hold at $20 \% \mathrm{~A} 80 \%$ $\mathrm{B}$, the composition was returned to $95 \% \mathrm{~A} 5 \% \mathrm{~B}$ ready for the next injection. Total UHPLC analysis time was 13 min per sample. All solvent gradients were linear. The injection volume was $0.5 \mu \mathrm{L}$. Spectral data $(260-600 \mathrm{~nm})$ were collected for the entire analysis, and the anthocyanin components were identified and quantified from chromatograms extracted at $530 \mathrm{~nm}$. External calibration curves were constructed for cyanidin 3-O-glucoside obtained from Extrasynthese, (Genay, France). All anthocyanin concentrations were calculated as cyanidin 3-O-glucoside equivalents on a fresh weight basis. Individual anthocyanins were identified based on similarity of retention times and spectral properties to authentic compounds (cyanidin 3-O-glucoside, cyanidin 3-O-galactoside) when available, or to anthocyanins isolated during previous research [33]. Chromatographic data were collected and manipulated using the Chromeleon ${ }^{\circledR}$ Chromatography Management System version 6.8 (Dionex Corporation).

\section{Statistical analysis}

We used 'heatmap.2' function of the 'gplots' package in $\mathrm{R}$ [40] to construct heatmaps of pigment data for two of the backcross populations with larger family sizes. A heatmap is a graphical display of data which uses colours to represent the numerical values. For example, smaller 
values can be assigned cooler blue tones while the larger values can tend towards hotter orange and red colours. Heatmaps are often used to encode and display multivariate data where several variables are measured on a set of entities (e.g. vines) so that the data structure is a matrix of columns and rows. The colours are automatically scaled for each variable of the data matrix, but this can be disabled if the data have already been normalised. For multivariate data heatmaps can also rearrange rows and columns so that similar entities and variables are grouped together and each is represented by a dendrogram.

\section{Additional files}

\section{Additional file 1: Pedigree of the four Actinidia families that show} segregation of petal colour. All the parents involved in creating the four $F_{2}$ backcross families that demonstrated segregation of petal colour were from two Actinidia taxa, A. chinensis var. chinensis and A. eriantha. The parents of each generation are shown. The female parent is indicated by a pink blaze, and the male parent by blue. A. eriantha contributed the red petal phenotype to the segregating population.

Additional file 2: Alleles of MYB110a carried by the parents of the four $F_{2}$ backcross Actinidia families. The alleles of MYB110a that are present in all parents of the backcross are represented by the sizes of the PCR products amplified by the primers of the marker Ke923. Where experimental data could not be generated, through loss of the parental genotype, the sizes of the alleles were inferred from the parents and progeny of that genotype.

Additional file 3: Red expression in petals, anther filaments and ovaries in the flowers of four F2 backcross hybrid Actinidia families. Ovary colour was independent of petal colour. Stamen filament colour was also independent of petal and ovary colour. Red filaments were found with both red or white petals, and red or green ovaries, in all combinations in female progeny, and with red or white petals in males.

Additional file 4: Anthocyanin and flavonoid analysis data. Identities and concentrations of anthocyanins and flavonols extracted from red petals of flowers of four inter-related $F_{2}$ backcross Actinidia families.

Additional file 5: Gene-specific primer sequences. Quantitative realtime PCR was carried out with gene-specific primers that were designed using Vector NTI 9.0.0. PCR primers of marker Ke923 specific for MYB110a, and marker Ke701 specific for MYB110b were designed by LGF.

\section{Competing interests}

We do not have any competing interests to report.

\section{Authors' contributions}

LGF conceived the genotyping study, carried out SSR discovery, marker polymorphism discovery and population genotyping and analyses, drafted the manuscript, and coordinated the final version. AGS carried out phenotyping, and discovered the 1:1 segregation ratio for flower colour in the backcross population. AGS and HEM bred the hybrid population. JKD assisted with phenotyping. GKT and EH participated in population genotyping; GKT also assisted in preparing the manuscript. PMD performed genotyping analyses and map construction. HNDS assisted in the design of the study and performed the statistical analysis, and wrote the statistical methods section. TKM carried out the quantification and identification of anthocyanins and flavonols and wrote the method for the manuscript. MM sequenced relevant portions of the genome, carried out qPCR, and assisted with manuscript preparation. ACA advised study directions, carried out transient assays and had input in manuscript preparation. KMD contributed to revision of the manuscript draft. MAM assisted with sample collection and manuscript preparation. All authors read and approved the final manuscript.

\section{Acknowledgements}

We wish to thank Tim Holmes, Darren Snaith and Minna Pesonen of Plant \& Food Research Design and Photography Group for photography and compilation of the figures. We also thank K Hoeata and B Hofstee and the Te Puke Research Centre orchard staff for propagating and maintaining the vines. This work was funded by the New Zealand Ministry of Science and Innovation contracts C11X1007 and CO6X0207.

\section{Author details}

'The New Zealand Institute for Plant \& Food Research Limited, 120 Mt. Albert Road, Auckland 1142, New Zealand. ${ }^{2}$ The New Zealand Institute for Plant \& Food Research Limited, 412 No. 1 Road, RD 2, Te Puke 3182, New Zealand. ${ }^{3}$ The New Zealand Institute for Plant \& Food Research Limited, Fitzherbert Science Centre, Batchelar Road, Palmerston North 4474, New Zealand.

Received: 11 June 2012 Accepted: 7 January 2013

Published: 16 January 2013

\section{References}

1. Gould K, Lister C: Flavonoid functions in plants. In Flavonoids: Chemistry, Biochemistry, and Applications. Edited by Andersen OM, Markham K. Boca Raton, Florida: CRC Press: 2006:397-411.

2. Grotewold E: The genetics and biochemistry of floral pigments. Annu Rev Plant Biol 2006, 57:761-780.

3. de Pascual-Teresa S, Sanchez-Ballesta MT: Anthocyanins: from plant to health. Phytochem Rev 2008, 7:281-299.

4. Kay CD: Aspects of anthocyanin absorption, metabolism and pharmacokinetics in humans. Nutr Res Rev 2006, 19:137-146.

5. Stevenson DE, Hurst RD: Polyphenolic phytochemicals - just antioxidants or much more? Cell Mol Life Sci 2007, 64:2900-2916.

6. Zafra-Stone S, Yasmin T, Bagchi M, Chatterjee A, Vinson JA, Bagchi D: Berry anthocyanins as novel antioxidants in human health and disease prevention. Mol Nutr Food Res 2007, 51:675-683.

7. Baudry A, Heim MA, Dubreucq B, Caboche M, Weisshaar B, Lepiniec L: TT2, $\Pi \pi$, and $\Pi \mathrm{G} 1$ synergistically specify the expression of BANYULS and proanthocyanidin biosynthesis in Arabidopsis thaliana. Plant J 2004, 39:366-380.

8. Koes R, Verweij W, Quattrocchio F: Flavonoids: a colorful model for the regulation and evolution of biochemical pathways. Trends Plant Sci 2005, 10:236-242.

9. Lepiniec L, Debeaujon I, Routaboul J-M, Baudry A, Pourcel L, Nesi N, Caboche M: Genetics and biochemistry of seed flavonoids. Annu Rev Plant Biol 2006, 57:405-430.

10. Aharoni A, De Vos CHR, Wein M, Sun ZK, Greco R, Kroon A, Mol JNM O'Connell AP: The strawberry FaMYB1 transcription factor suppresses anthocyanin and flavonol accumulation in transgenic tobacco. Plant 2001, 28:319-332.

11. Albert NW, Lewis DH, Zhang H, Schwinn KE, Jameson PE, Davies KM: Members of an R2R3-MYB transcription factor family in Petunia are developmentally and environmentally regulated to control complex floral and vegetative pigmentation patterning. Plant J 2011, 65:771-784.

12. Dubos C, Le Gourrierec J, Baudry A, Huep G, Lanet E, Debeaujon I, Routaboul J-M, Alboresi A, Weisshaar B, Lepiniec L: MYBL2 is a new regulator of flavonoid biosynthesis in Arabidopsis thaliana. Plant J 2008, 55:940-953

13. Lin-Wang K, Micheletti D, Palmer J, Volz R, Lozano L, Espley R, Hellens RP, Chagne D, Rowan DD, Troggio $M$, et al: High temperature reduces apple fruit colour via modulation of the anthocyanin regulatory complex. Plant Cell Environ 2011, 34:1176-1190.

14. Matsui K, Umemura Y, Ohme-Takagi M: AtMYBL2, a protein with a single MYB domain, acts as a negative regulator of anthocyanin biosynthesis in Arabidopsis. Plant J 2008, 55:954-967.

15. Allan AC, Hellens RP, Laing WA: MYB transcription factors that colour our fruit. Trends Plant Sci 2008, 13:99-102.

16. Schwinn K, Venail J, Shang YJ, Mackay S, Alm V, Butelli E, Oyama R, Bailey P, Davies $\mathrm{K}$, Martin C: A small family of MYB-regulatory genes controls floral pigmentation intensity and patterning in the genus Antirrhinum. Plant Cell 2006, 18:831-851.

17. Feller A, Machemer K, Braun EL, Grotewold E: Evolutionary and comparative analysis of MYB and bHLH plant transcription factors. Plant J 2011, 66:94-116. 
18. Gonzalez A, Zhao M, Leavitt JM, Lloyd AM: Regulation of the anthocyanin biosynthetic pathway by the $\Pi \mathrm{TG} 1 / \mathrm{bHLH} / \mathrm{Myb}$ transcriptional complex in Arabidopsis seedlings. Plant J 2008, 53:814-827

19. Martin C, Ellis N, Rook F: Do transcription factors play special roles in adaptive variation? Plant Physiol 2010, 154:506-511.

20. Quattrocchio F, Wing J, van der Woude K, Souer E, de Vetten N, Mol J, Koes R: Molecular analysis of the anthocyanin2 gene of petunia and its role in the evolution of flower color. Plant Cell 1999, 11:1433-1444.

21. Cui Z, Huang H, Xiao X: Actinidia in China. Beijing: China Agricultural Science and Technology Press; 2002.

22. Wang SM, Huang HW, Jiang ZW, Zhang ZH, Zhang SR, Huang HQ: Studies on Actinidia breeding by species hybridization between $A$. chinensis and A. eriantha and their hybrids' progenies. In Advances in Actinidia Research. Edited by Huang HW.; 2000:126-132.

23. Xiong ZT, Wang SM, Huang RH: Studies on the hybridization between Actinidia chinensis var. chinensis and A. eriantha. Journal of Wuhan Botanical Research 1987, 5:321-328.

24. Zhang L, Li Z, Wang Y, Jiang Z, Wang S, Huang H: Vitamin C, flower color and ploidy variation of hybrids from a ploidy-unbalanced Actinidia interspecific cross and SSR characterization. Euphytica 2010, 175:133-143.

25. Fan P, An H, Cai D, Mu X, Fan PG, An HX, Cai DR, Mu XJ: Interspecific hybridization between species of Actinidia $L$. and breeding of superior selection. Journal of Fruit Science 2004, 21:208-211.

26. Crowhurst RN, Gleave AP, MacRae EA, Ampomah-Dwamena C, Atkinson RG, Beuning LL, Bulley SM, Chagne D, Marsh KB, Matich AJ, et al: Analysis of expressed sequence tags from Actinidia: Applications of a cross species EST database for gene discovery in the areas of flavor, health, color and ripening. BMC Genomics 2008, 9:351.

27. Lin-Wang K, Bolitho K, Grafton K, Kortstee A, Karunairetnam S, McGhie TK, Espley RV, Hellens RP, Allan AC: An R2R3 MYB transcription factor associated with regulation of the anthocyanin biosynthetic pathway in Rosaceae. BMC Plant Biol 2010, 10:50.

28. Zimmermann IM, Heim MA, Weisshaar B, Uhrig JF: Comprehensive identification of Arabidopsis thaliana MYB transcription factors interacting with R/B-like BHLH proteins. Plant J 2004, 40:22-34.

29. Grotewold E, Sainz MB, Tagliani L, Hernandez JM, Bowen B, Chandler VL: Identification of the residues in the Myb domain of maize $\mathrm{C} 1$ that specify the interaction with the bHLH cofactor R. Proc Natl Acad Sci U S A 2000, 97:13579-13584.

30. Fraser LG, Tsang GK, Datson PM, De Silva HN, Harvey CF, Gill GP, Crowhurst RN, McNeilage MA: A gene-rich linkage map in the dioecious species Actinidia chinensis (kiwifruit) reveals putative $X / Y$ sex-determining chromosomes. BMC Genomics 2009, 10:102.

31. Montefiori M, Espley RV, Stevenson D, Cooney J, Datson PM, Saiz A, Atkinson RG, Hellens RP, Allan AC: Identification and characterisation of F3GT1 and F3GGT1, two glycosyltransferases responsible for anthocyanin biosynthesis in red-fleshed kiwifruit (Actinidia chinensis). Plant J 2011, 65:106-118

32. Espley RV, Hellens RP, Putterill J, Stevenson DE, Kutty-Amma S, Allan AC: Red colouration in apple fruit is due to the activity of the MYB transcription factor, MdMYB10. Plant J 2007, 49:414-427.

33. Comeskey DJ, Montefiori M, Edwards PJB, McGhie TK: Isolation and structural identification of the anthocyanin components of red kiwifruit. J Agric Food Chem 2009, 57:2035-2039.

34. Montefiori M, Comeskey DJ, Wohlers M, McGhie TK: Characterization and quantification of anthocyanins in red kiwifruit (Actinidia spp.). J Agric Food Chem 2009, 57:6856-6861.

35. Castaneda-Ovando A, de Lourdes P-HM, Elena Paez-Hernandez M, Rodriguez JA, Andres Galan-Vidal C: Chemical studies of anthocyanins: A review. Food Chem 2009, 113:859-871.

36. Davies AJ, Mazza G: Copigmentation of simple and acylated anthocyanins with colorless phenolic compounds. J Agric Food Chem 1993, 41:716-720.

37. Johnson ET, Yi HK, Shin BC, Oh BJ, Cheong HS, Choi G: Cymbidium hybrida dihydroflavonol 4-reductase does not efficiently reduce dihydrokaempferol to produce orange pelargonidin-type anthocyanins. Plant J 1999, 19:81-85.

38. Kumar S, Tamura K, Nei M: MEGA3: Integrated software for molecular evolutionary genetics analysis and sequence alignment. Brief Bioinform 2004, 5:150-163.
39. Hellens RP, Allan AC, Friel EN, Bolitho K, Grafton K, Templeton MD, Karunairetnam S, Gleave AP, Laing WA: Transient expression vectors for functional genomics, quantification of promoter activity and RNA silencing in plants. Plant methods 2005, 1:13.

40. Gplots: Various $R$ programming tools for plotting data. $R$ package version 2.10.0. http://CRAN.Rproject.org/package=gplots.

doi:10.1186/1471-2164-14-28

Cite this article as: Fraser et al:: An R2R3 MYB transcription factor determines red petal colour in an Actinidia (kiwifruit) hybrid population. BMC Genomics 2013 14:28.

\section{Submit your next manuscript to BioMed Central and take full advantage of:}

- Convenient online submission

- Thorough peer review

- No space constraints or color figure charges

- Immediate publication on acceptance

- Inclusion in PubMed, CAS, Scopus and Google Scholar

- Research which is freely available for redistribution 